\title{
Logarithmic Image Processing for Color images
}

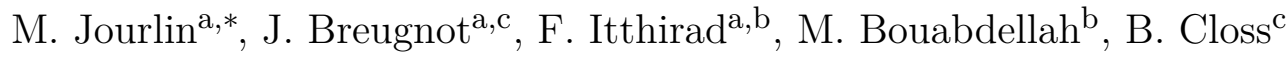 \\ ${ }^{a}$ University of Saint-Etienne, Lab. H. Curien UMR CNRS 5516, Saint-Etienne, France \\ ${ }^{b}$ NT2I, 20 Rue B. Lauras, 42000 Saint-Etienne, France \\ ${ }^{c}$ Silab, Z.I. de la Nau, 19240 Saint-Viance, France
}

\begin{abstract}
The LIP (Logarithmic Image Processing) framework is classically devoted to grayscale images. The aim of the present study is to extend this framework to color images. This new model is noted LIPC for LIP Color. It does not consist in applying the LIP Model to each channel R, G, B of a color image. We define the transmittance of color images in order to give a physical justification, on which will be based the definition of logarithmic operators like addition, subtraction and scalar multiplication, respectively noted in the LIPC : $\triangle_{c}, \triangle_{c}$ and $\otimes_{c}$.

As for the classical LIP Model, the laws ${\AA_{c}}_{c}$ and $\otimes_{c}$ define a vector space structure on the space of images which enables us to present notions requiring such a structure. For example, we define a color logarithmic interpolation by associating to a pair $(\mathbf{F}, \mathbf{G})$ of images the interval $[\mathbf{F}, \mathbf{G}]$, set of barycenters of $\mathbf{F}$ and $\mathbf{G}$. A new notion of color contrast is defined, which satisfies sub-additivity and homogeneity for scalar multiplication. This notion is proved to be efficient for edge detection.

We note that the vector space structure opens the way to a lot of developments concerning the definition of metrics, norms, scalar products...and to transfer to LIPC gauges theory, duality theory...

In this initial paper, we preferred insist on applications of the LIPC. For example, color prediction is presented and discussed as well as stabilization of images by dynamic range centring and enhancement of under-lighted images. Concerning the implementation of the LIPC operators and algorithms, informations are given on their execution time.
\end{abstract}

Keywords: logarithmic image processing, image enhancement, color images, human vision 


\section{Contents}

\begin{tabular}{lll}
\hline & Introduction & 3
\end{tabular}

1.1 The LIP Model framework . . . . . . . . . . . . . . . . . . . 3

1.2 Color image processing . . . . . . . . . . . . . . . . . . 7

$\begin{array}{|ll|}2 & \text { Logarithmic Image Processing for Color Images }\end{array}$

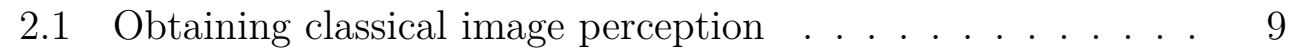

2.2 Obtaining transmittance perception . . . . . . . . . . . . . 11

2.3 Internal addition on the set $\mathcal{I}^{3} \ldots \ldots \ldots \ldots$

2.4 External scalar multiplication . . . . . . . . . . . . . . . . 14

2.5 Subtraction of two images . . . . . . . . . . . . . . . . . . . 14

2.6 Transmittance operation's properties . . . . . . . . . . . . . 15

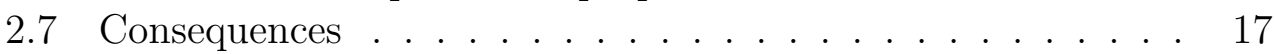

2.8 Direct Applications $\quad \ldots \ldots \ldots$

2.8 .1 Addition . . . . . . . . . . . . . . . . . . . . 19

$2.8 .2 \quad$ Multiplication . . . . . . . . . . . . . . . . . . . . . 19

2.8 .3 Subtraction . . . . . . . . . . . . . . . . . . . 20

2.8 .4 Interpolation $\ldots \ldots \ldots \ldots$. . . . . . . . . . . 20

2.9 Color prediction $\ldots \ldots \ldots \ldots$

2.10 Contrast definition . . . . . . . . . . . . . . . . . . . 21

2.11 Enhancement and stabilization . . . . . . . . . . . . . 23

2.11 .1 Optimal multiplication factor . . . . . . . . . . 23

2.11 .2 Fixed mean value . . . . . . . . . . . . . . . . . . 24

2.11 .3 Enhancement . . . . . . . . . . . . . . . . . 24

2.12 Implementation . . . . . . . . . . . . . . . . . . 26

\begin{tabular}{lll}
\hline 3 & Conclusion & 28
\end{tabular}

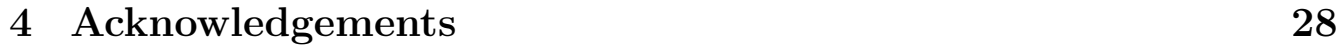

$\begin{array}{lll}5 & \text { Bibliography } & 28\end{array}$

\begin{tabular}{lll}
\hline Main notations & 31
\end{tabular}

* Corresponding author

Email addresses: michel.jourlin@univ-st-etienne.fr (M. Jourlin ), josselin.breugnot@univ-st-etienne.fr (J. Breugnot), f.itthirad@nt2i.fr (F. Itthirad ), m.bouabdellah@nt2i.fr (M. Bouabdellah), b.closs@silab.fr (B. Closs) 
\begin{tabular}{lll}
\hline 7 & Appendices & 31
\end{tabular}

\begin{tabular}{|l|l|}
\hline A Image multiplication demonstration & 31
\end{tabular}

B Associativity of LIPC addition 33

C Distributivity of scalar addition with respect to LIPC multiplication

D Distributivity of LIPC scalar multiplication with respect to LIPC addition 34

E Associativity of LIPC multiplication 34

F Ten degrees RGB Color Matching Function of Stiles and Burch (1959) 35

\begin{tabular}{lll}
\hline G D65 power spectral distribution & 37
\end{tabular}

\begin{tabular}{ll}
\hline H Figures & 39
\end{tabular}

\section{Introduction}

\subsection{The LIP Model framework}

This part is devoted to recalls on the grey level LIP Model. At the very beginning of the LIP Model, M. Jourlin in Jourlin and Pinoli (1985) put forward this model as a mathematical framework where the addition of two grey level functions $F^{\text {" }+" ~} G$ could be possible.

Mathematicians are familiar with the idea that the addition of two functions is generally driven by the addition of the arrival space. In fact, given a set $E$ without any structure and two functions $F$ and $G$ defined on it, with values for example in the real space $\mathbb{R}$, an addition of $F$ and $G$ is immediately available :

$$
\forall x \in E, \quad\left(F^{"}+" G\right)(x)=F(x)+G(x)
$$

In the case of images defined on a same spatial support $D \subset \mathbb{R}^{2}$ and with values in the grey-scale [0,M[, such an approach is not possible because the grey levels are bounded and consequently the addition of two grey levels 
would not always stay in the scale. Those remarks have caused some authors to "truncate" the addition in order to limitate its values to the grey scale.

Coming back to the LIP construction, M. Jourlin decided to try to find a physical framework where the addition of two grey level functions remains a grey level function. It appears that the case of images acquired in transmission (i.e. when the observed scene is situated between the source and the sensor) corresponds to this situation, because the physical addition of two semi-transparent objects produces an image. Thus, the addition $F \bowtie G$ of two images defined on $D \subset \mathbb{R}^{2}$ with values in $[0, M[$ is now possible thanks to the transmittance law :

$$
T_{F \triangleleft G}=T_{F} \times T_{G}
$$

and thanks to the link between $T_{F}$ and the function $F$ itself (see Jourlin and Pinoli (2001)) :

$$
T_{F}=1-\frac{F}{M}
$$

Here, the transmittance $T_{F}(x)$ at a point $x$ of $D$ represents the probability for an element of the source incident at $x$ to "pass through" the object, i.e. to be seen by the sensor. In physical terms :

$$
T_{F}(x)=\frac{\Phi_{x}^{O}}{\Phi_{x}^{I}}
$$

where $\Phi_{x}^{O}$ designs the out-coming flux at $x$ for $\mathrm{F}$, and $\Phi_{x}^{I}$ designs the incoming flux at each point of $D$, noted $\Phi^{I}$ when this flux is supposed homogeneous (i.e. presenting the same value at each $x \in D$ ). Using relations (2) in formula (1) yields to :

$$
F \nrightarrow G=F+G-\frac{F G}{M}
$$

which appears as an internal addition on the space $\mathcal{I}(D,[0, M[)$ of images defined on $D$ with values in $[0, M[$, (cf figure 1$)$.

From this addition, a scalar multiplication has been derived, starting of 
the particular situation where we add the same object to itself.

$$
F \AA F=2 F-\frac{F^{2}}{M} \text { that we can write }: 2 \rtimes F
$$

The formula has been generalized to $n \otimes F$ for each integer $n \in \mathbb{N}$, then to each quotient of integers $\frac{p}{q}$ and finally to each real number $\alpha$ according to :

$$
\alpha \bowtie F=M-M\left(1-\frac{F}{M}\right)^{\alpha}
$$

M. Jourlin suggested that J.C. Pinoli works with him on the development of this LIP Model, in the context of a $\mathrm{PhD}$ thesis, which resulted in presenting the basic properties of the LIP Model through various initial publications : Jourlin and Pinoli (1988) and Jourlin et al. (1989), and later in Jourlin and Pinoli (1995). For the reader interested in more details, we recommend a synthesis of further properties and applications in Jourlin and Pinoli (2001). They concern the characterization of $(\mathcal{I}(D,[0, M[), \AA, \otimes)$ as the positive cone of the vector space $(\mathcal{F}(D]-,\infty, M[), \AA, \otimes)$ of all functions defined on $D$ with values in $]-\infty, M[$.

From this vectorial structure, many mathematical tools are derived, like interpolation (in a logarithmic sense), metrics, correlation and a scalar product by Pinoli (1992), as well as an algorithm of stabilization of a signal (see Jourlin and Pinoli (1995)) and a regression model in the previously defined vector space (see Pumo and Dhorne (1998)).

An important property of the LIP Model has been established by Brailean et al. (1991) : the LIP Model is consistent with human vision and thus is applicable on images for which the processing must approach the human perceptual system, even when these images are acquired in a situation of reflected light. Furthermore, a large number of materials observed in reflection are semi-transparent: human skin, polymers, varnishes, paints, cosmetic products... In all these cases, the interaction "light-material" must take into account a non-linear attenuation of the source intensity through the object. Such a remark considerably enlarges the Model's field of interest.

Now let us consider the possibility of extending the LIP Model to color images. A preliminary unrefined approach consists in processing each channel of a RGB image in a logarithmic manner. It produces some interesting 
results but it does not take into account the different sensitivities of the human eye in the Red, Green and Blue channels, and can produce false colors (see Garcia (2008) and figure 2).

Some previous studies have put forward solutions to perform color logarithmic processing. An example is the model of V. Patrascu described in Patrascu (2001) in his PhD thesis directed by V. Buzuloiu, Patrascu and Buzuloiu (2001a), Patrascu and Buzuloiu (2001b), Patrascu and Buzuloiu (2002), Patrascu and Buzuloiu (2003a), Patrascu and Buzuloiu (2003b). The adopted solution consists in considering the interval $[-1,+1]$ as the grey scale and $[-1,+1]^{3}$ as the color scale. Thanks to this presentation, the vector structure is easy to define, but in our appreciation, the physical justification of the model is not established. The "Romanian school" directed by V. Buzuloiu has developed works on this model by Florea et al. (2007) and Zaharescu (2003).

Other solutions have been proposed, especially by Liévin and Luthon (2004) and Luthon et al. (2010). They created the LUX (for Logarithmic hUe eXtension) Color System according to the following definitions :

$$
\begin{aligned}
& L=(R+1)^{0.3}(G+1)^{0.6}(B+1)^{0.1}-1 \\
& U=\left\{\begin{array}{c}
128\left(\frac{L+1}{R+1}\right) \text { if } R>L \\
256-128\left(\frac{R+1}{L+1}\right) \text { otherwise }
\end{array}\right. \\
& X=\left\{\begin{array}{c}
128\left(\frac{L+1}{B+1}\right) \text { if } B>L \\
256-128\left(\frac{B+1}{L+1}\right) \text { otherwise }
\end{array}\right.
\end{aligned}
$$

and applied it with success in speech recognition for lips segmentation.

We propose in this paper a model called LIPC (for LIP Color), that uses the eye wavelength sensitivity. It will be demonstrated that this model is clearly mathematically and physically defined taking into account the sensitivity of the eye and its perception of colors, associated to different operators as in the LIP Model.

Fundamental remark : Since the LIP model uses images acquired in transmission, the observed object is placed between the sensor and the lighting 
source. Let's assume there is no object between the source and the sensor. The image we get will be completely white. If a layer is added, the image from the sensor will be darker since there is a semi-transparent object in front of the source. However, by definition, the result should remain unchanged if the neutral element is added. Thus, the neutral element corresponds to the value 0 and is the brightest grey level (white).

By adding an infinite number of objects, the sensor may not receive light anymore and the image will be completely black. Therefore, the grey scale $[0, \mathrm{M}[$ has been chosen where 0 is the neutral element of the addition law. It is the reason why it was called the "white" extremity (cf figure 3). The other extremity $\mathrm{M}$ is generally equal to $2^{8}=256$.

This point of view presents the advantage that in the extended space, noted $\mathcal{F}(D]-,\infty, M[)$, the negative values correspond to opposite values of "true" grey levels lying in $[0, M[$.

On the opposite, this choice does not correspond to the "classical" grey scale $[0, M[$ where 0 is the black extremity. Nevertheless, it is easy to adapt the LIP Model to this context by inversion of the grey-scale, that is to say by replacing the value $F(x)$ by $M-F(x)$. Thus, the grey scale becomes ] $0, M]$ where 0 corresponds now to the black pixels. A translation of one unit $(F(x)$ replaced by $M-F(x)-1)$ yields to the current interval [0, $M[$. Under such conditions, we must take care that the addition neutral element becomes 255 .

In this case, the over space $\mathcal{F}(D]-,\infty, M[)$ becomes $\mathcal{F}(D] 0,,+\infty[)$, where the values $[0, M[$ are the "true" grey values corresponding to images and the interval $[M,+\infty$ [ represents the opposite values of $[0, M[$.

\subsection{Color image processing}

A color image represents multi-spectral data acquired through the visible domain. For "classical" color images, this domain is reduced in three channels, one for each primary color : red, green and blue. A simple way to process those images is to use the same algorithm on each channel (see figure (4). Nevertheless, this method doesn't take into account the correlation between the channels. Another approach consists in processing the entire data at the same time (see figure 5). 
In the example on figure 6, we apply a histogram equalization to image "Lena" using those 2 approaches. With the first one, the red, green and blue channels are well equalized separately but false colors appear. Hues are preserved in the second one.

Some studies propose to classify colors in order to maintain the data correlation. A lexicographic order consists for example to define the red color "inferior" to the blue one because the letter $\mathrm{R}$ is placed after the letter B in the alphabet. This concept is useful when an order relation is absolutely necessary, like in the color mathematical morphology.

One solution to process color images is to change the color space. The aim is to find a space where data are not correlated. The color space CIELAB 1931 has been created in order to represent our visual system behaviour. It separates the luminance and the chrominance informations.

Therefore, common algorithms "adapted to the human vision" only work on the luminance channel in the $\mathrm{L}^{*} \mathrm{a}^{*} \mathrm{~b}^{*}$ space (see figure 8). Thus, false colors can't be generated.

\section{Logarithmic Image Processing for Color Images}

In the next sections we differentiate LIP (Logarithmic Image Processing for grey level images) and LIPC (Logarithmic Image Processing for Color images).

Based on the LIP framework properties, LIPC must be mathematically and physically justified, as well as consistent with human vision.

To define the transmittance of a color image, we use the same transmitted signal approach than the one used for grey level LIP. A scene is typically acquired through a camera with a Bayer filter or a 3-CCD sensor. The image is then defined with Red, Green and Blue values and the display device may be an 8 bits computer screen with the same 3 channels.

A color image is defined as a function $\mathbf{F}$ on a spatial support $D \subset \mathbb{R}^{2}$ with 3 components $F r, F g, F b$ taking values in $[0,255]$ for each channel $R, G, B$.

In this context, our color transmittance is linked to two models : on one 
hand, the ocular perception of an image on a computer screen, and on the other, the ocular perception of a transmittance (thanks to a light source placed behind the semi-transparent layer).

Furthermore, in order to keep the human perceptual system approach, we take into account the eye sensitivity in the visible domain by using a Color Matching Functions table (see next section).

It will be demonstrated how the situation described in the precedent fundamental remark may be exactly adapted to the LIPC Model with 3 color components lying in $\left[0, M\left[\right.\right.$ and giving for a 24 bits images the space $\mathcal{I}^{3}\left(D,[0,255]^{3}\right)$ or $\mathcal{I}^{3}$ when no confusion is possible.

Mathematically, the over-space $\mathcal{F}^{3}\left(D,\left[0,+\infty\left[{ }^{3}\right)\right.\right.$ becomes a vector space for the addition law $\AA_{c}$ and the multiplication law $\otimes_{c}$. The space of images $\mathcal{I}^{3}$ represents its positive cone and the values in $\left[M,+\infty\left[^{3}\right.\right.$ the opposite of real color values lying in $[0,255]^{3}$.

Remark : Our color images are defined in the RGB space. In the case of multi-spectral data on more than 3 channels, the model can be easily adapted. We will develop this point in a further publication.

\subsection{Obtaining classical image perception}

Initially, we define how the eye perceives an image shown by a display unit (cf (a) in figure 7). The eye is similar to a sensor called $\mathbf{S}$ (with 3 channels $S_{R}, S_{G}$ and $\left.S_{B}\right)$ and the perception $\mathbf{P}$ takes account of the observed image $\mathbf{F} \in \mathcal{I}^{3}$ and the light source $\mathbf{L}$.

The sensor is divided into numerous cells, as many as the image pixels number. Each is divided into 3 channels (R, G and B) and receives part of the light coming from the image. Subsequently, $P_{i}$ (with $i \in[R, G, B]$ ) can be defined as the sum along the visible spectrum of these different elements.

Given a cell $(x, y)$, we define :

$$
P_{i}(x, y)=\int_{\lambda=390}^{780} L(\lambda) F_{i}(x, y) S_{i}(\lambda) d \lambda \quad i \in[R, G, B]
$$


Thus, the light coming from the image to the sensor, comes actually from the 3 primaries of the display unit $R=700 \mathrm{~nm}, G=545 \mathrm{~nm}$ and $B=435 \mathrm{~nm}$. Thereby, the light source is separated into 3 components according to the primaries, and balanced by some constants $C_{R}, C_{G}, C_{B}$, depending on the sensor sensitivity. In order to display a white sensation to the observer, a certain amount of each primary has to be emitted, in relation with the eye wavelength sensitivity.

From experiments on 49 observers, Stiles and Burch have measured this sensitivity and created a table called Color Matching Functions (CMF, cf tables in appendix $\mathrm{F}$. The observers had to reproduce a monochromatic color by merging R, G, B colors. The CMF describe the eye $(\mathbf{S})$ behaviour on values from 390 to $780 \mathrm{~nm}$ every $5 \mathrm{~nm}$. The negative values (figure 9 ) correspond to the unreproducible colors which require adding a certain amount of a component (see Schanda (2007)).

$$
C_{R}=\frac{1}{S_{R}\left(\lambda_{R}\right)}, C_{G}=\frac{1}{S_{G}\left(\lambda_{G}\right)}, C_{B}=\frac{1}{S_{B}\left(\lambda_{B}\right)}
$$

We note :

$$
L_{R}=C_{R} L(700), L_{G}=C_{G} L(545), L_{B}=C_{B} L(435)
$$

Under a matrix formulation, relation (5) can be written :

$$
\left(\begin{array}{c}
P_{R} \\
P_{G} \\
P_{B}
\end{array}\right)=\left(\begin{array}{lll}
L_{R} S_{R}\left(\lambda_{R}\right) & L_{G} S_{R}\left(\lambda_{G}\right) & L_{B} S_{R}\left(\lambda_{B}\right) \\
L_{R} S_{G}\left(\lambda_{R}\right) & L_{G} S_{G}\left(\lambda_{G}\right) & L_{B} S_{G}\left(\lambda_{B}\right) \\
L_{R} S_{B}\left(\lambda_{R}\right) & L_{G} S_{B}\left(\lambda_{G}\right) & L_{B} S_{B}\left(\lambda_{B}\right)
\end{array}\right)\left(\begin{array}{c}
F_{R} \\
F_{G} \\
F_{B}
\end{array}\right)
$$

and then :

$$
\mathbf{P}=\mathbf{K F}
$$

The result has to be bounded in order to stay within the definition domain. When the $\mathbf{K}$ matrix is applied to an image whose values are equal (when normalized by the dynamic range) to the unit vector $\mathbf{N}=[1,1,1]$, the normalized result (by $M$ ) of the operation $\mathbf{K N}$ must be equal to $\mathbf{N}$. We introduce the matrix $\mathbf{X}$ which allows us to obtain $(\mathbf{K N}) * \mathbf{X}=\mathbf{N}$ where $*$ 
represents the "element by element" multiplication.

$$
\mathbf{X}=\left(\begin{array}{c}
X_{R} \\
X_{G} \\
X_{B}
\end{array}\right)=\left(\begin{array}{c}
\frac{1}{L_{R} S_{R}\left(\lambda_{R}\right)+L_{G} S_{R}\left(\lambda_{G}\right)+L_{B} S_{R}\left(\lambda_{B}\right)} \\
\frac{1}{L_{R} S_{G}\left(\lambda_{R}\right)+L_{G} S_{G}\left(\lambda_{G}\right)+L_{B} S_{G}\left(\lambda_{B}\right)} \\
\frac{1}{L_{R} S_{B}\left(\lambda_{R}\right)+L_{G} S_{B}\left(\lambda_{G}\right)+L_{B} S_{B}\left(\lambda_{B}\right)}
\end{array}\right)
$$

We note $\mathbf{K}$ the result of $\mathbf{K} * \mathbf{X}$

$$
\dot{\mathbf{K}}=\left(\begin{array}{ccc}
X_{R} L_{R} S_{R}\left(\lambda_{R}\right) & X_{R} L_{G} S_{R}\left(\lambda_{G}\right) & X_{R} L_{B} S_{R}\left(\lambda_{B}\right) \\
X_{G} L_{R} S_{G}\left(\lambda_{R}\right) & X_{G} L_{G} S_{G}\left(\lambda_{G}\right) & X_{G} L_{B} S_{G}\left(\lambda_{B}\right) \\
X_{B} L_{R} S_{B}\left(\lambda_{R}\right) & X_{B} L_{G} S_{B}\left(\lambda_{G}\right) & X_{B} L_{B} S_{B}\left(\lambda_{B}\right)
\end{array}\right)
$$

Finally we can express the sensor perception matrix of a classical image, solely with the image data and the $\mathbf{K}$ matrix :

$$
\mathbf{P}=\mathbf{K}\left(\begin{array}{c}
F_{R} \\
F_{G} \\
F_{B}
\end{array}\right)
$$

\subsection{Obtaining transmittance perception}

In order to describe the transmittance $\mathbf{T}$ of an image $\mathbf{F} \in \mathcal{I}^{3}$, some elements have to be described :

- $T(\lambda, x, y)$ is the wavelength distribution of the transmittance, depending on the position $(x, y)$, which will be noted $T(\lambda)$. Given the CMF values, we can divide the visible domain into three parts $T_{390 \rightarrow 480}$, $T_{485 \rightarrow 545}$ and $T_{550 \rightarrow 780}$ where the blue, green and red colors are respectively dominant. It gives the matrix representation :

$$
\mathbf{T}=\left(\begin{array}{l}
T_{390 \rightarrow 480} \\
T_{485 \rightarrow 545} \\
T_{550 \rightarrow 780}
\end{array}\right)
$$

- $L(\lambda)$ : wavelength distribution of the lighting source,

- $S_{i}(\lambda)$ : sensor wavelength distribution depending on the channel $i \in$ $\{R, G, B\}$, in our case the eye.

Now let us assume the following hypotheses : 
- the lighting source $L(\lambda)$ is uniform on the spatial support which is a reasonable hypothesis according to disposable light sources

- the transmittance $\mathbf{T}$ is piecewise continuous

Under such conditions, the sensor perception is defined as the integration along the visible spectrum of the light source spectrum, the sensor sensitivity and the transmittance (see (b) on figure 7). Each channel of the sensor perception takes values in $[0,255]$ and a transmittance is classically defined on the interval $[0,1]$. So, the transmittance has to be multiplied by the dynamic range $M$ to correspond to the values of $\mathbf{P}$ previously defined in (6).

$$
P_{i}=M \int_{390}^{780} L(\lambda) T(\lambda) S_{i}(\lambda) d \lambda
$$

As $T(\lambda)$ is piecewise continuous as said in the hypotheses, it can be extracted out of the integration :

$$
P_{i}=M \sum_{j=390}^{780} T_{j} \int_{j}^{j+1} L(\lambda) S_{i}(\lambda) d \lambda
$$

where each interval $(j, j+1)$ corresponds to a continuity domain of $T$. The right part of the expression is noted :

$$
U_{i, j}=M \int_{j}^{j+1} L(\lambda) S_{i}(\lambda) d \lambda
$$

and the corresponding matrix $\mathbf{U}$ can be reduced, merging the wavelength domain into 3 parts :

$$
\mathbf{U}=M\left(\begin{array}{ccc}
U_{R, 390 \rightarrow 480} & U_{R, 485 \rightarrow 545} & U_{R, 550 \rightarrow 780} \\
U_{G, 390 \rightarrow 480} & U_{G, 485 \rightarrow 545} & U_{G, 550 \rightarrow 780} \\
U_{B, 390 \rightarrow 480} & U_{B, 485 \rightarrow 545} & U_{B, 550 \rightarrow 780}
\end{array}\right)
$$

As we want the result of the multiplication of $\mathbf{U}$ by $\mathbf{T}$ to take values in $[0,1]$, given a transmittance $\mathbf{T}$ with values equal to the unit vector $\mathbf{N}$, the 
result of $\mathbf{U} * \mathbf{T}$ must be $\mathbf{N} ;(\mathbf{U N}) * \mathbf{Y}=\mathbf{N}$.

$$
\Leftrightarrow \mathbf{Y}=\left(\begin{array}{l}
Y_{R} \\
Y_{G} \\
Y_{B}
\end{array}\right)=\frac{\mathbf{N}}{\mathbf{U N}}=\frac{1}{\mathbf{U}}=\left(\begin{array}{c}
\frac{1}{\sum_{j=390}^{780} U_{R, j}} \\
\frac{1}{\sum_{j=390}^{780} U_{G, j}} \\
\frac{1}{\sum_{j=390}^{780} U_{B, j}}
\end{array}\right)
$$

With the wavelength domain merged as previously, the outcoming matrix of perception $\mathbf{P}$ may be expressed as :

$$
\mathbf{P}=\mathbf{U} \mathbf{T} \text { where } \mathbf{U}=\mathbf{U} *\left(\begin{array}{c}
Y_{R} \\
Y_{G} \\
Y_{B}
\end{array}\right)
$$

Thanks to CMF and D65 illuminant values, Ú can be easily computed (see equation 14 for values). As $\operatorname{det}(\mathbf{U}) \neq 0$, the matrix Ú can be inverted :

$$
\mathbf{T}=\mathbf{U}^{-1} \mathbf{P}
$$

Thanks to relation (6), the definition of $\mathbf{P}$ is well known, which gives us the expression of $\mathbf{T}$ depending exclusively on the image $\mathbf{F}$ and the matrices $\mathbf{U}$ and $\mathbf{K}$ :

$$
\mathbf{T}=\mathbf{U}^{-1} \mathbf{K} \mathbf{F}
$$

\subsection{Internal addition on the set $\mathcal{I}^{3}$}

In this section we will define the theoretical laws (internal addition, external multiplication) in the space $\mathcal{I}^{3}$ of images : in the following sections, the LIPC addition will be denoted by the symbol $\AA_{c}$. In the LIP framework and more generally in the transmitted signal processing, the transmittance of the addition of two images $\mathbf{F} \in \mathcal{I}^{3}$, and $\mathbf{G} \in \mathcal{I}^{3}$ is equivalent to the multiplication of their transmittances $\mathbf{T}_{\mathbf{F}}$ and $\mathbf{T}_{\mathbf{G}}$ according to :

$$
\mathbf{T}_{\mathbf{F}_{\Perp_{c}} \mathbf{G}}=\mathbf{T}_{\mathbf{F}} * \mathbf{T}_{\mathbf{G}}
$$


In 2.2 we have defined that $\mathbf{P}=\mathbf{U} \mathbf{T}$; for each pairs of images $\mathbf{F}, \mathbf{G} \in \mathcal{I}^{3}$

$$
\mathbf{P}_{\mathbf{F}\lrcorner_{c} \mathbf{G}}=\mathbf{U}\left(\mathbf{T}_{\mathbf{F}} * \mathbf{T}_{\mathbf{G}}\right)
$$

We replace $\mathbf{T}_{\mathbf{F}}, \mathbf{T}_{\mathbf{G}}$ and $\mathbf{P}_{\mathbf{F} A_{c} \mathbf{G}}$ by their relative expressions thanks to relations (9) and (6) and we obtain :

$$
\begin{gathered}
\hat{\mathbf{K}}\left(\mathbf{F} \star_{c} \mathbf{G}\right)=\mathbf{U}^{\prime}\left(\mathbf{U}^{-1} \dot{\mathbf{K}} \mathbf{F} * \mathbf{U}^{-1} \mathbf{K} \mathbf{G}\right) \\
\left.\Leftrightarrow \mathbf{F} \star_{c} \mathbf{G}=\dot{\mathbf{K}}^{-1} \mathbf{U}^{-\mathbf{U}^{-1}} \mathbf{K} \mathbf{F} * \dot{\mathbf{U}}^{-1} \mathbf{K} . \mathbf{G}\right)
\end{gathered}
$$

As said previously, Ḱ can be inverted thanks to the values computed from CMF and D65 (see formula 15).

\subsection{External scalar multiplication}

The symbol $\otimes_{c}$ denotes the external LIPC multiplication by a scalar $\alpha \in \mathbb{R}$. We start from the addition of an image onto itself in the LIPC framework. For $\mathbf{F} \in \mathcal{I}^{3}$ :

$$
\begin{aligned}
\mathbf{F} \star_{C} \mathbf{F} & =\dot{\mathbf{K}}^{-1} \mathbf{U}\left(\mathbf{U}^{-1} \mathbf{K} \mathbf{F} * \mathbf{U}^{-1} \mathbf{K} . \mathbf{F}\right) \\
\Leftrightarrow 2 \widehat{\otimes}_{c} \mathbf{F} & =\mathbf{K}^{-1} \mathbf{U}^{\prime}\left(\mathbf{U}^{-1} \mathbf{K} \mathbf{F}\right)^{2}
\end{aligned}
$$

We repeat the operation, making the addition between the image $\mathbf{F}$ and the result of $2 \otimes_{C} \mathbf{F}$ :

$$
\begin{aligned}
\mathbf{F} \triangle_{c}\left(2 \otimes_{c} \mathbf{F}\right) & =3 \otimes_{c} \mathbf{F} \\
3 \otimes_{c} \mathbf{F} & =\dot{\mathbf{K}}^{-1} \mathbf{U}^{\prime}\left(\mathbf{U}^{-1} \mathbf{K} \mathbf{F} * \mathbf{U}^{-1} \mathbf{K}^{\prime}{ }^{-1} \mathbf{U}^{\prime}\left(\mathbf{U}^{-1} \mathbf{K} \mathbf{F}\right)^{2}\right) \\
3 \otimes_{c} \mathbf{F} & =\mathbf{K}^{-1} \mathbf{U}^{\prime}\left(\mathbf{U}^{-1} \mathbf{K} \mathbf{F}\right)^{3}
\end{aligned}
$$

By recurrence, we prove in appendix $\mathrm{A}$ that for each $n \in \mathbb{N}$ :

$$
n \otimes_{c} \mathbf{F}=\dot{\mathbf{K}}^{-1} \mathbf{U}^{\prime}\left(\mathbf{U}^{-1} \mathbf{K} \mathbf{F}\right)^{n}
$$

Then, it is generalized to $\alpha \in \mathbb{R}$ in the same appendix $\mathrm{A}$.

\subsection{Subtraction of two images}

The symbol $\triangle_{c}$ denotes the LIPC subtraction. The subtraction is a combination of the two previous operators addition and multiplication. 
Given $\mathbf{F}$ and $\mathbf{G} \in \mathcal{I}^{3}$ :

$$
\mathbf{F} \triangle_{c}\left(-1 \widehat{\triangle}_{c} \mathbf{G}\right)=\mathbf{F} \triangle_{c} \mathbf{G}
$$

Replacing the corresponding expressions gives :

$$
\left.\mathbf{F} \triangle_{c} \mathbf{G}=\mathbf{U}^{-1} \mathbf{K}\left(\mathbf{U}^{-1} \mathbf{K} \mathbf{F} * \mathbf{U}^{-1} \mathbf{K}^{-1} \mathbf{U}^{-\mathbf{U}^{-1}} \mathbf{K} \mathbf{G}\right)^{-1}\right)
$$

After simplification :

$$
\mathbf{F} \triangle_{c} \mathbf{G}=\dot{\mathbf{K}}^{-1} \mathbf{U}\left(\frac{\mathbf{U}^{-1} \hat{\mathbf{K}} \mathbf{F}}{\mathbf{U}^{-1} \hat{\mathbf{K}} \mathbf{G}}\right)
$$

A condition for being compatible with the mathematical definition is that values of $\mathbf{F}$ must be superior to $\mathbf{G}$, otherwise the result is not in the definition domain $[0,255]^{3}$ but in $\left[0,+\infty\left[^{3}\right.\right.$. We could also take into account only positive values to bring the result back to the definition domain, paying attention that the result is truncated, generating a loss of information.

2.6. Transmittance operation's properties

Let us demonstrate some properties of the previous operations :

- Associativity of LIPC addition (see appendix B)

$\forall \mathbf{F}, \mathbf{G}, \mathbf{H} \in \mathcal{I}^{3}$

$$
\mathbf{F} \star_{c}\left(\mathbf{G} \star_{C} \mathbf{H}\right)=\left(\mathbf{F} \star_{c} \mathbf{G}\right) \oplus_{c} \mathbf{H}
$$

- Commutativity of LIPC addition

Each pair of images $\mathbf{F}, \mathbf{G}$ satisfies :

$$
\mathbf{F} \AA_{c} \mathbf{G}=\mathbf{G} \AA_{c} \mathbf{F}
$$

because of :

$$
\mathbf{U}^{-1} \mathbf{K} \mathbf{F} * \mathbf{U}^{-1} \mathbf{K} . \mathbf{G}=\mathbf{U}^{-1} \mathbf{K} \mathbf{G} * \mathbf{U}^{-1} \mathbf{K} . \mathbf{F}
$$

- Neutral element of LIPC addition 
We can show that there exists an image $\mathbf{G}_{0}$, assimilated to a white transparent layer, whose values are equal to the vector $[255,255,255]$ (for 24 bits color images), such that for each $\mathbf{F} \in \mathcal{I}^{3}$ :

$$
\mathbf{F} \AA_{c} \mathbf{G}_{0}=\mathbf{F}
$$

- Opposite element of LIPC addition

We define for each $\mathbf{F} \in \mathcal{I}^{3}$ its opposite element $\triangle_{c} \mathbf{F}$, such that :

$$
\mathbf{F} \triangle_{c}\left(\triangle_{c} \mathbf{F}\right)=\mathbf{G}_{0}
$$

because :

$$
\mathbf{F} \triangle_{c}\left(\triangle_{c} \mathbf{F}\right)=\mathbf{K}^{-1} \mathbf{U}^{\prime}\left(\mathbf{U}^{-1} \mathbf{K} \mathbf{F} * \mathbf{U}^{-1} \mathbf{K}\left(\triangle_{c} \mathbf{F}\right)\right)=\mathbf{G}_{0}
$$

and

$$
\mathbf{U}^{-1} \mathbf{K} \mathbf{G}_{0}=\mathbf{N}
$$

We show that:

$$
\triangle_{c} \mathbf{F}=\dot{\mathbf{K}}^{-1} \mathbf{U}^{\prime} *\left(\frac{1}{\mathbf{U}^{-1} \mathbf{K}_{\mathbf{F}}}\right)
$$

Remark : such a function $\triangle_{c} \mathbf{F}$ has been mathematically defined, but does not lie in the space $\mathcal{I}^{3}$ of images. In fact, it takes values in $\left[255,+\infty\left[^{3}\right.\right.$. For this reason, we will define an "over-space" on $\mathcal{I}^{3}$, noted $\mathcal{F}^{3}\left(D,\left[0,+\infty\left[{ }^{3}\right)\right.\right.$, or $\mathcal{F}^{3}$.

At this step, we can conclude that $\mathcal{F}^{3}$ equipped with the law $\AA_{c}$ presents all the properties of an additive group.

Moreover, concerning the scalar multiplication, $\left(\mathcal{F}^{3}, \otimes_{c}\right)$ satisfies:

- Distributivity of scalar addition with respect to LIPC multiplication (cf appendix C)

$\forall \alpha, \beta \in \mathbb{R}^{+}, \forall \mathbf{F} \in \mathcal{I}^{3}$

$$
(\alpha+\beta) \otimes_{c} \mathbf{F}=\left(\alpha \otimes_{c} \mathbf{F}\right) \AA_{c}\left(\beta \otimes_{c} \mathbf{F}\right)
$$


- Distributivity of LIPC scalar multiplication with respect to LIPC addition (cf appendix D)

$\forall \alpha \in \mathbb{R}^{+}, \forall \mathbf{F}, \mathbf{G} \in \mathcal{I}^{3}$

$$
\left.\alpha \otimes_{c}\left(\mathbf{F} \star_{c} \mathbf{G}\right)=\left(\alpha \otimes_{c} \mathbf{F}\right) \star_{c} \alpha \otimes_{c} \mathbf{G}\right)
$$

- Associativity of LIPC multiplication (cf appendix E)

$$
\forall \alpha, \beta \in \mathbb{R}^{+}, \forall \mathbf{F} \in \mathcal{I}^{3}
$$

$$
\alpha \otimes_{c}\left(\beta \otimes_{c} \mathbf{F}\right)=(\alpha \times \beta) \otimes_{c} \mathbf{F}
$$

- Existence of a neutral element for LIPC Multiplication

For each $\mathbf{F} \in \mathcal{I}^{3}$ there exists a neutral element noted $\mathbf{1}$ such that :

$$
\mathbf{1} \otimes_{c} \mathbf{F}=\hat{\mathbf{K}}^{-1} \mathbf{U}^{\prime}\left(\mathbf{U}^{-1} \mathbf{K} \mathbf{F}\right)^{1}=\mathbf{F}
$$

\subsection{Consequences}

Thanks to the previous properties, one can afford that:

$\left(\mathcal{F}^{3}, \triangle_{c}, \otimes_{c}\right)$ is a real vector space (in fact a vector space defined on $[0,+\infty[$ )

and the space of images $\left(\mathcal{I}^{3}, \AA_{c}, \otimes_{c}\right)$ represents its positive cone.

Fundamental Remark : before presenting some applications of LIPC Model in the next section, we ask the reader to pay attention to the following points :

- considering the space $\left(\mathcal{I}^{3}, \triangle_{c}, \widehat{\otimes}_{c}\right)$ is not a vector space itself does not really limit the interest of such a strong structure : in fact, the behaviour of $\left(\mathcal{I}^{3}, \triangle_{c}, \otimes_{c}\right)$ related to the vector space $\left(\mathcal{F}^{3}, \Delta_{c}, \otimes_{c}\right)$ is exactly comparable to that of $\left(\mathbb{R}^{+}\right)^{2}$ in $\mathbb{R}^{2}$ : two elements lying in the positive cone are elements (vectors) of the vector space. Thus, the numerous properties established by mathematicians in the context of vector spaces or topological vector spaces (see Grothendieck (1954), Michael (1952) and Bourbaki (2006)) are available in the positive cone, if they do not necessitate the use of "opposite" 
vectors or multiplication by a "negative" scalar (in fact lying in $[M,+\infty[$ ). As examples, we can mention the notions of interpolation between two images (presented below), scalar product, distances...

- Furthermore, the vector space structure opens very important transfers from mathematics to image processing : duality theory, gauges theory (development in progress). The possibility to define topologies compatible with the vector structure (i.e. such that the laws $\Delta_{c}$ and $\Delta_{c}$ be continuous) will allow to characterize some properties of operators defined on $\left(\mathcal{I}^{3}, \AA_{c}, \otimes_{c}\right)$ with values in $\left(\mathcal{I}^{3}, \AA_{c}, \widehat{\triangle}_{c}\right)$, which is the most common situation of image transforms. As an example, it is very important (but rarely studied) to precise if an image processing operator is continuous or not (does it preserve the proximity of resulting images if the distance between initial ones is small ?).

- A weak utilisation of the LIPC model consists in translating classical operators (gradient, Laplacian, metrics...) into "logarithmic" ones. Generally, such a transcription does not present difficulties and then is of no interest at a theoretical level. Nevertheless, it is often very useful because the LIPC Model is consistent with human vision : in each situation of image processing where we aim at imitating the human behaviour, the use of $\triangle_{c}, \Delta_{c}$ and $\triangle_{c}$ is efficient. The same situation was observed in the LIP context : Deng et al. (1995), Deng and Cahill (1993), Deng (2009), Deng and Pinoli (1998) and Panetta et al. (2008), for example have defined gradient notions in a logarithmic way producing a better visual detection and a more precise one in the dark parts of images.

- Let us insist on the fact that, at the beginning, the LIP Model was adapted to images acquired in transmitted signal. In such situations the use of LIPC operators will be also very efficient (see 2.9p).

- Finally, considering that on one hand, the operators $\triangle_{c}$ and $\triangle_{c}$ result in darkening or lightening a given image, and that $\alpha \Delta_{c}$ simulates the thickness increasing $(\alpha>1)$ or decreasing $(\alpha<1)$ of the semi-transparent obstacle generating the image, it is evident that such operators are very well adapted to process :

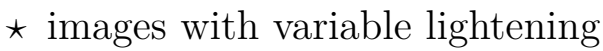

* images acquired under low-lightening or very low lightening near night 
vision

A special part of the paper will be devoted to that specific kind of applications.

\subsection{Direct Applications}

\subsubsection{Addition}

We choose the D65 illuminant, which is generally sufficient for common images. The $\mathbf{K}$ and Ú matrices are computed once and for all, thanks to the CMF tables given in appendix F, D65 tables in appendix G and formula (7).

$$
\mathbf{U}=\left(\begin{array}{ccc}
25,0440 & 53,1416 & 176,8144 \\
21,3002 & 185,9744 & 47,7254 \\
229,2474 & 19,9944 & 5,7583
\end{array}\right)
$$

and

$$
\mathbf{K}=\left(\begin{array}{lll}
0.6991 & 0.2109 & 0.0899 \\
0.1947 & 0.8002 & 0.0049 \\
0.0681 & 0.0002 & 0.9315
\end{array}\right)
$$

Unlike the classical addition between two images, LIPC addition does not exceed the bounds of the grey scale for each $R, G$ and $B$ channel.

The operation behaves as a superposition of two semitransparent layers taking account of subtractive synthesis. Colors and contrasts of the two initial images (figure 12) are well preserved without visible false color generation.

\subsubsection{Multiplication}

The LIPC multiplication by a scalar allows darkening or brightening of an image, depending on the value of the scalar. If the scalar $\in] 0,1[$, the multiplication proceeds as a suppression of layers and the result is brighter than the original image.

Conversely, using a scalar $\alpha \in] 1 ;+\infty[$ will return a darker image, corresponding to the superposition of the image on itself $\alpha$ times.

One can see on the result (figure 13) that shadows are well upgraded, without saturation of brighter areas. The result matches the initial colors. 


\subsubsection{Subtraction}

The LIPC framework enables a subtraction of non diffusing images. Given two images $\mathbf{F}$ and $\mathbf{G}$, the result of LIPC addition of $\mathbf{F}$ and $\mathbf{G}$ then subtracted by $\mathbf{G}$ (using LIPC subtraction), should be the original image $\mathbf{F}$. Using classical operators, the result differs markedly from the original image.

On the result (image (d) on figure 14), we can see that with LIPC operations, the result is the same image as the initial one. The subtraction operator is also able to suppress the background layer from an image.

As a real condition test, we have decomposed a color image into two complementary layers. For each pixel's color, we are able to find two RGB values whose LIPC addition gives that same initial color. One application of this method is color prediction, described in 2.9. As you can see on figure 18, the result of the LIPC addition of our decomposition perfectly fits the original image.

\subsubsection{Interpolation}

We can define the LIPC interpolation between 2 images. In fact, this notion is classically associated to a vector space structure (or a positive cone of a vector space). The interpolation between 2 images $\mathbf{F}$ and $\mathbf{G} \in \mathcal{I}^{3}$ is easily obtained by combination of LIPC addition and LIPC multiplication according to :

$$
[\mathbf{F}, \mathbf{G}]=\left\{\left(\lambda \otimes_{c} \mathbf{F}\right) \triangle_{c}\left[(1-\lambda) \otimes_{c} \mathbf{G}\right]\right\}_{\lambda \in[0,1]}
$$

where $[\mathbf{F}, \mathbf{G}]$ designs the "segment" generated by $\mathbf{F}$ and $\mathbf{G}$, i.e. the set of interpolated images.

One can see in figure 19 that transition made by LIPC operations perfectly reproduces the behaviour of transparent slides on each others. However, since the LIPC framework was initially made for low values, we may have better results for dark images than bright ones.

Such image interpolations were successfully used in the LIP context in various situations like :

- starting with the "physical" cuts of a 3D cell, if some cut lacks for any reason (bad cutting) it is possible to replace it by the interpolation (with 
$\lambda=\frac{1}{2}$ ) of the previous and next images (see Gremillet et al. (1991) and Gremillet et al. (1994)).

- when using images acquired with X-ray scanner, the (x,y) resolution (pixel size) inside a cut is generally much more precise than the z-resolution (voxel thickness). This fact produces voxels 4 or 5 times higher than large. In order to obtain voxels nearer to a cube shape, it is possible to create 3 or 4 interpolated images between two successive ones.

Such applications must be performed in the same manner for 3D color images (as for The Visible Human Project for example, see Ackerman (1994)).

\subsection{Color prediction}

Inspired from works of Hébert and Hersch (2006), Hébert and Hersch (2009) and Hébert and Hersch (2011), we have tried and succeed to perform one kind of color prediction. Given a random RGB color with values on $[0,255]^{3}$, we can define this color as a LIPC addition of two colors. Let's assume that our target color has $\mathrm{Rv}, \mathrm{Gv}$ and $\mathrm{Bv}$ values. The first color must be chosen in $([R v, 255],[G v, 255],[B v, 255])$ in a supervised or random manner. The second color is then given by the LIPC subtraction of the target color and our first color.

An example is given on figure 11, where we associate to a given set of random colors (figure 11 (a)) a complementary set of colors (figure 11 (b)) that visually gives the same result for each couple of colors (figure 11 (c) and $(d))$ : the first example aims to obtain a grey color whereas the second one, using the same initial set, aims to obtain a red color.

We note that in the case of a target color with a high predominance of a primary color, the complementary color will conserve the predominance.

\subsection{Contrast definition}

On a grey-scale image $F$, the contrast definition between two pixels $x$ and $y$ is derived from the physicians's one as :

$$
C_{x, y}(F)=\frac{\operatorname{Max}(F(x), F(y))-\operatorname{Min}(F(x), F(y))}{\operatorname{Max}(F(x), F(y))+\operatorname{Min}(F(x), F(y))}=\frac{|F(x)-F(y)|}{F(x)+F(y)}
$$

which clearly lies in $[0,1]$. Thus, it is obvious that in order to be displayed on a screen, contrast result has to be normalized between 0 and 255. Thanks 
to this definition, low grey levels values are favoured by comparison to the high ones.

A LIP version of contrast, noted $C_{x, y}^{\triangle}(F)$ has been proposed by Jourlin et al. (1989). In their approach $C_{x, y}^{\triangle}(F)$ represents the grey level which must be added to the Min in order to obtain the Max :

$$
C_{x, y}^{\triangle}(F)=\operatorname{Max}(F(x), F(y)) \triangle \operatorname{Min}(F(x), F(y))=\frac{|F(x)-F(y)|}{1-\frac{\operatorname{Min}(F(x), F(y)}{M}}
$$

Another approach using LIP Laplacian of Gaussian has been described by Palomares et al. (2005).

This contrast, as well as the physicians's one, links the perceptive approach of LIP Model. It respects some useful mathematics properties as the scalar LIP multiplication homogeneity $\left(C_{x, y}^{\triangle}(\lambda \otimes F)=\lambda 凶 C_{x, y}^{\triangle}(F)\right)$ and the subadditivity $\left(C_{x, y}^{\triangle}(F \triangleleft G) \leq C_{x, y}^{\triangle}(F) \wedge C_{x, y}^{\triangle}(G)\right)$. Contrast can be computed for two pixels of a same image or more generally for two images, by averaging the contrast between $F(x)$ and $G(x)$ where $x$ is lying in the definition domain $D$ or in a Region Of Interest of it. We propose a definition of a color contrast using LIPC framework limited to the comparison of a point to its neighbours.

Color contrast of a point of an image is defined thanks to his neighbours. Given an image $\mathbf{F}$ with $\mathrm{R}, \mathrm{G}, \mathrm{B}$ channels $F_{R}, F_{G}, F_{B}$, and a pixel $x$ of this image, each pixel $N_{i}$ of the eight pixels of the $N_{8}$ neighbourhood will be tested in order to evaluate which one has the largest contrast thanks to a LIPC subtraction. In order to avoid out of scale values, we shall not subtract lower values to the current one, which implies LIPC subtraction is computed as follows :

$$
\operatorname{Sub}(\mathbf{F}, x, i)=\left(\begin{array}{c}
\min \left(F_{R}(x), F_{R}\left(N_{i}\right)\right) \\
\min \left(F_{G}(x), F_{G}\left(N_{i}\right)\right) \\
\min \left(F_{B}(x), F_{B}\left(N_{i}\right)\right)
\end{array}\right) \Delta_{c}\left(\begin{array}{c}
\max \left(F_{R}(x), F_{R}\left(N_{i}\right)\right) \\
\max \left(F_{G}(x), F_{G}\left(N_{i}\right)\right) \\
\max \left(F_{B}(x), F_{B}\left(N_{i}\right)\right)
\end{array}\right)
$$

In the same manner as the LIP contrast, where the greatest difference between two pixels is actually the lowest value (i.e. the darkest layer), the greatest difference for a LIPC contrast will be the minimum norm of color 
values. Pixel contrast value can be assimilated to the subtraction value which gives the minimum norm. A LIPC contrast can be defined by selecting among the 8 following values :

$$
C_{x}^{\triangle_{c}}=\operatorname{Sub}(\mathbf{F}, x, j), j \in[1,8]
$$

that presenting a minimum norm :

$$
\begin{array}{r}
\|S u b(\mathbf{F}, x, j)\|=\min _{i=1 \ldots 8}\|S u b(\mathbf{F}, x, i)\| \\
\text { where } \forall x \in \mathbb{R},\|x\|=\sqrt{x^{2}}
\end{array}
$$

We chose to apply our contrast to the peppers' image. On homogeneous areas, the contrast is low but we can still discern the objects' colors (see figure 15). High contrasts are visible for each pepper's borders. Thus, one application of this color contrast may be edge detection. By applying a linear darkening on the image, high contrasts on edges remain and the result stays quite similar, which proves the efficiency of this approach on dark images or images with variable lightening.

\subsection{Enhancement and stabilization}

\subsubsection{Optimal multiplication factor}

A dynamic centring for color images is possible by transposing a LIP method described by Jourlin and Pinoli (1995). This algorithm suggests an optimal factor $\lambda_{0}$ to enhance the dynamic range.

$\lambda_{0}$ is given by : $\lambda_{0}=\frac{\ln \left(\frac{\ln \left(1-\frac{f(a)}{M}\right)}{\ln \left(1-\frac{f(b)}{M}\right)}\right)}{\ln \left(\frac{1-\frac{f(b)}{M}}{1-\frac{f(a)}{M}}\right)}$

with $F(a)$ the maximum of the grey levels of the image and $F(b)$ the minimum of the grey levels of the image.

We extend this method to color images by searching the maximum and minimum values of grey levels over the 3 channels. For an image $\mathbf{F} \in \mathcal{I}^{3}$ the 
enhanced image is given by $\lambda_{0} \otimes_{c} \mathbf{F}$, cf fig 20 .

\subsubsection{Fixed mean value}

Another approach aims at a stabilization of images acquired under variable lightening simulated by variable aperture values of the sensor objective. It consists in applying a LIPC operation in order to maintain the mean value at a desired level (125 for example, cf figure 22).

The darker the image, the more scattered the histogram will be, due to the fact that dark images have a few grey levels and that mean centring does not create new levels, but only expands them. Another result is given for example in figure 21 .

\subsubsection{Enhancement}

First let us propose an efficient approach dedicated to low-light images. It consists on applying to the image a subtraction by an adequate constant $C$ in order to expand the dynamic range to the whole scale (grey or color).

- Concerning grayscale images (LIP framework)

If $F$ denotes an underlighted image, it presents a small dynamic range $d r(F)$, concentrated near the black extremety $M$ of the grey scale $[0, M[$.

There exists $a$ and $b$ lying in $D$ where $F$ reaches respectively its maximal and minimal values:

$$
F(a)=\operatorname{Sup}_{x \in D} F(x) \quad F(b)=\operatorname{In} f_{x \in D} F(x)
$$

Thus $a$ and $b$ represent the darkest and the brightest points of $D$ for the image $F$ and we have :

$$
d r(F)=F(a)-F(b)
$$

Given some grey level $C$, let us compute the values $F(a) \triangle C$ and $F(b) \triangle C$ which may be negative ones if $C>F(a)$.

The dynamic range of the image $F \triangle C$ is given by :

$$
d r(F \triangle C)=F(a) \triangle C-F(b) \triangle C=\frac{F(a)-C}{1-\frac{C}{M}}-\frac{F(b)-C}{1-\frac{C}{M}}=\frac{F(a)-F(b)}{1-\frac{C}{M}}
$$

Remark : because $1-\frac{C}{M}<1$ for each $\left.C \in\right] 0, M[$, the dynamic range $d r(F \triangle C)$ is greater than $d r(F)$, and tends to infinity when $C$ tends to $M$. For 8 bits 
images $(M=256)$, we propose to compute the value $C_{0}$ of $C$ satisfying $d r\left(f \triangle C_{0}\right)=255$ :

$$
\frac{F(a)-F(b)}{1-\frac{C_{0}}{M}}=255 \Leftrightarrow C_{0}=M\left[1-\frac{F(a)-F(b)}{M}\right]
$$

It remains only to translate $F \triangle C_{0}$ by the value $-F(b) \triangle C_{0}$ in order to display it as an image.

- Concerning color images (LIPC framework)

The same approach is possible. If $\mathbf{F}$ represents now a color image $\left(F_{R}, F_{G}, F_{B}\right)$, we compute :

$$
\operatorname{Sup}(\mathbf{F})=\operatorname{Sup}_{x \in D}\left(F_{R}(x), F_{G}(x), F_{B}(x)\right)
$$

and

$$
\operatorname{Inf}(\mathbf{F})=\operatorname{In} f_{x \in D}\left(F_{R}(x), F_{G}(x), F_{B}(x)\right)
$$

Considering the dynamic range $d r(\mathbf{F})$ as the difference $\operatorname{Sup}(\mathbf{F})-\operatorname{In} f(\mathbf{F})$ and the fact that these two values lying in the interval $[0, M[$ (where 0 is the "black value" in the LIPC model), one can apply the same expansion than for a grey level image, which gives a unique value $\mathbf{C}_{0}$ defined by searching :

$$
\begin{gathered}
d r\left(\mathbf{F} \triangle_{c} \mathbf{C}_{0}\right)=\left(\mathbf{A} \triangle_{c} \mathbf{C}_{0}\right)-\left(\mathbf{B} \triangle_{c} \mathbf{C}_{0}\right)=\operatorname{Max} \\
\text { where } \mathbf{A}=\left(\begin{array}{c}
\operatorname{Sup}(\mathbf{F}) \\
\operatorname{Sup}(\mathbf{F}) \\
\operatorname{Sup}(\mathbf{F})
\end{array}\right), \mathbf{B}=\left(\begin{array}{c}
\operatorname{Inf}(\mathbf{F}) \\
\operatorname{Inf}(\mathbf{F}) \\
\operatorname{Inf}(\mathbf{F})
\end{array}\right) \text { and } \mathbf{M a x}=\left(\begin{array}{c}
255 \\
255 \\
255
\end{array}\right)
\end{gathered}
$$

$\mathbf{C}_{0}$ expression is given by :

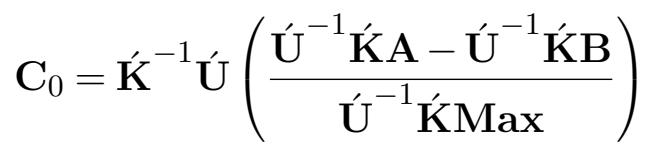

Finally $\mathbf{C}_{0}$ which values are $\left(\begin{array}{c}C_{0} \\ C_{0} \\ C_{0}\end{array}\right)$, is subtracted to $\mathbf{F}=\left(\begin{array}{c}F_{R} \\ F_{G} \\ F_{B}\end{array}\right)$ and the $\operatorname{result}\left(\begin{array}{c}F_{R} \\ F_{G} \\ F_{B}\end{array}\right) \triangle_{c}\left(\begin{array}{c}C_{0} \\ C_{0} \\ C_{0}\end{array}\right)$ is translated by $-\operatorname{Inf}\left[\left(\begin{array}{c}F_{R} \\ F_{G} \\ F_{B}\end{array}\right) \triangle_{c}\left(\begin{array}{c}C_{0} \\ C_{0} \\ C_{0}\end{array}\right)\right]$ in 
order to be displayed (see figure 16).

Not many grey levels are employed in images acquired with low lightening conditions. The greatest value of each channel is far from the maximum. It is as if a dark semi-transparent image had been superposed on a bright one. Enhancement can be approached by LIPC subtraction, by subtracting a grey value, which value depends on the maximum of the three channels. If the image minimum is 0 , as it is commonly in dark scenes, the subtraction brings a maximum dynamic to the new image. An example is given in figure 17 where one can see how improved is the image.

This method necessitates an important condition : bright dots must not appear on the image, otherwise, since maximum is already high, enhancement could be limited. Another approach consists on subtracting a grey value, which is equivalent as to subtract a grey semi-transparent image. This method was used in the case of outdoors acquisition with low lightning conditions. Using a common Bayer camera, we have taken a scene at two different exposure times: 30 and 100ms. 20 to 40 lux were measured under the street lamp, and approximatively 0.5 lux in dark areas. Obviously, images are brighter with a higher exposure time. We have compared LIPC subtraction with the highest camera's gain correction and with histogram equalization. It results that gain compensation is not enough and that saturation areas appear with the equalization. With our algorithm, more details are visible and the level of correction can be chosen (see figure 23).

\subsection{Implementation}

The execution time depends on the operation and on the image size. We choose two images to illustrate the execution time of our algorithms. One is the well known Lena $(512 \times 512)$, the other is a natural scene with bigger dimensions (1920x1080) (see figure 10). The Hardware configuration is composed by a Core i7 860 @ 2.80GHZ, with NVIDIA Quadro 2000 (192 multiprocessors).

For Matlab users, we compared a basic implementation and an improvement using Mex (C language for Matlab). For $\mathrm{C}++$ developers, we compared a basic implementation and an improvement using NVIDIA CUDA's parallel programming model. 
For both images, there is a significant gain when using accelerations. We note that Mex and $\mathrm{C}++$ are quite similar. By using GPU computing, it is even possible to do real-time color image processing in the sense of acquisition speed (25 images per second).

Remark : Complex values can appear when performing LIPC multiplication and one must only keep real values. It is automatically done by Matlab. For classical LIP operations, a speed-up is possible by using lookup tables. It isn't possible for LIPC as it would take too much memory. Furthermore, it may be faster to process the image directly than calculating the LUT and applying it.

\begin{tabular}{|c|c|c|c|}
\hline Operation & $\hat{A}_{c}$ & $\widehat{A}_{c}$ & $\widehat{\triangle}_{c}$ \\
\hline execution time Matlab (ms) & 3453 & 5991 & 3778 \\
\hline execution time Mex (ms) & 31 & 32 & 233 \\
\hline execution time C++ (ms) & 19 & 25 & 88 \\
\hline execution time CUDA (ms) & 0,59 & 0,66 & 0,6 \\
\hline
\end{tabular}

Table 1: execution time depending on language used on image lena (size 512x512 pixels see (a) on figure 10 )

\begin{tabular}{|c|c|c|c|}
\hline Operation & $\hat{+}_{c}$ & $\widehat{\Delta}_{c}$ & $\widehat{x}_{c}$ \\
\hline execution time Matlab (ms) & 26967 & 48086 & 28799 \\
\hline execution time Mex (ms) & 203 & 219 & 1641 \\
\hline execution time C++ (ms) & 157 & 198 & 1736 \\
\hline execution time CUDA (ms) & 3,69 & 3,96 & 3,76 \\
\hline
\end{tabular}

Table 2: execution time depending on language used on image P110 (size 1920x1080 pixels see (b) on figure 10) 


\section{Conclusion}

With adaptation of grey level Logarithmic Image Processing to color, we provide new kinds of tools for manipulating color images within the framework of a mathematically and physically justified model. These techniques are well adapted for human perception thanks to the use of color matching functions of the human eye in the elaboration of the model parameters. We note that our results depend on the precision of the color matching function we use, and that diffusion and reflection phenomena are not yet taken into account.

Moreover, some significant improvement has been realized (cf section 2.12) considering algorithm execution time, demonstrating that real time treatments can be performed.

\section{Acknowledgements}

The authors thank Dr. P. Hawkes, Editor of Advances in Imaging and Electron Physics, for his help and encouragement to publish this contribution to color image processing.

Many thanks are also due to : Johan Aubert, Mathieu Forichon and especially Stephane Grousson, who strongly contributed to the emergence of LIPC when they were students of Dr. Michel Jourlin.

\section{Bibliography}

Ackerman, Michael, J., 1994. The us national library of medicine's visible human project data sets.

Bourbaki, 2006. Espaces Vectoriels Topologiques.

Brailean, J., Sullivan, B., Chen, C., Giger, M., 1991. Evaluating the em algorithm for image processing using a human visual fidelity criterion. In: Proceedings of the International Conference on Acoustics. Speech and Signal Processing, pp. 2957-2960.

Deng, G., 2009. An entropy interpretation of the logarithmic image processing model with application to contrast enhancement. IEEE Transactions on Image Processing $18(5), 1135-1140$.

Deng, G., Cahill, L., 1993. Multiscale image enhancement using the logarithmic image processing model. Electronics Letters 29 (9), 803-804.

Deng, G., Cahill, L., Tobin, G., 1995. The study of logarithmic image processing model and its application to image enhancement. IEEE Transactions on Image Processing $4(4), 506-512$. 
Deng, G., Pinoli, J., 1998. Differentiation-based edge detection using the logarithmic image processing model. Journal of Mathematical Imaging and Vision 8, 161- 180 .

Florea, C., Vertan, C., Florea, L., 2007. Logarithmic model-based dynamic range enhancement of hip x-ray images. Proceedings of Advanced Concepts for Intelligent Vision Systems, 587- -596 .

Garcia, A., 06 2008. Analyse statistique et morphologique des images multivaluées : développements logiciels pour les applications cliniques. Ph.D. thesis, Ecole Normale Supérieure de CACHAN.

Gremillet, P., Jourlin, M., Bron, C., Schupbach, J., Gautschi, H., 1991. Dedicated image analysis techniques for three-dimensionnal reconstruction from serial sections in electron microscopy. Machine Vision and Applications 4 (4), 263-270.

Gremillet, P., Jourlin, M., Pinoli, J., 1994. Lip model based three-dimensionnal reconstruction and visualization of hiv-infected entire cells. Journal of Microscopy 174 (1), $31-38$.

Grothendieck, A., 1954. Espaces vectoriels topologiques. Sociedade de mathematica de São Paulo.

Hébert, M., Hersch, R. D., Oct 2006. Reflectance and transmittance model for recto-verso halftone prints. J. Opt. Soc. Am. A 23 (10), 2415-2432.

Hébert, M., Hersch, R. D., Feb 2009. Reflectance and transmittance model for rectoverso halftone prints: spectral predictions with multi-ink halftones. J. Opt. Soc. Am. A 26 (2), 356-364.

Hébert, M., Hersch, R. D., 2011. Yule-nielsen based recto-verso color halftone transmittance prediction model. JAppl. Opt.

Jourlin, M., Pinoli, J.-C., Décembre 1985. A model for logarithmic image processing. Tech. Rep. 3, Université de Saint-Etienne,Département de Mathématiques.

Jourlin, M., Pinoli, J.-C., 1988. A model for logarithmic image processing. J. Microsc. $149,21-35$.

Jourlin, M., Pinoli, J.-C., 1995. Image dynamic range enhancement and stabilization in the context of the logarithmic image processing model. Signal Process. 41 (2), 225-237.

Jourlin, M., Pinoli, J.-C., 2001. The mathematical and physical framework for the representation and processing of transmitted images. Advances in imaging and electron physics $115,129-196$.

Jourlin, M., Pinoli, J.-C., Zeboudj, R., 1989. Contrast definition and contour detection for logarithmic images. J. Microsc 156, 33-40.

Liévin, M., Luthon, F., 2004. Nonlinear color space and spatiotemporal mrf for hierarchical segmentation of face features in video. IEEE Trans Image Process 13 (1), $63-71$.

Luthon, F., Beaumesnil, B., Dubois, N., 2010. Lux color transform for mosaic image rendering. International Conference on Automation, Quality and Testing, Robotics 3, $1-6$.

Martin, D., Fowlkes, C., Tal, D., Malik, J., July 2001. A database of human segmented natural images and its application to evaluating segmentation algorithms and measuring ecological statistics. In: Proc. 8th Int'l Conf. Computer Vision. Vol. 2. pp. 416-423.

Michael, E., 1952. Transformations from a linear space with weak topology. Proceedings of the American Mathematical Society. 
Palomares, J., González, J., Ros, E., 2005. Designing a fast convolution under the lip paradigm applied to edge detection. Pattern Recognition and Image Analysis, Lecture Notes in Computer Science 3687, 560-569.

Panetta, Karen, A., Wharton, Eric, J., Agaian, Sos, S., 2008. Logarithmic edge detection with applications. Journal of Computers 3 (9), 11-19.

Patrascu, V., 2001. A mathematical model for logarithmic image processing. Ph.D. thesis, "Politehnica" University of Bucharest.

Patrascu, V., Buzuloiu, V., 2001a. Color image enhancement in the framework of logarithmic models. In: The 8Pthp IEEE International Conference on Telecommunications, Bucharest, Romania. Vol. 1. pp. 199-204.

Patrascu, V., Buzuloiu, V., 2001b. A mathematical model for logarithmic image processing. In: The 5PthP World Multi-Conference on Systemics, Cybernetics and Informatics, Orlando, USA. Vol. 13. pp. 117-122.

Patrascu, V., Buzuloiu, V., 2002. The affine transforms for image enhancement in the context of logarithmic models. In: Proceedings of International Conference on Computer Vision and Graphics, Zakopane, Poland. Vol. 2. pp. 596-601.

Patrascu, V., Buzuloiu, V., 2003a. Color correction method using logarithmic operations. In: Proceedings of the 7PthP International Conference on Engineering of Modern Electric Systems, Oradea, Romania. pp. 150-155.

Patrascu, V., Buzuloiu, V., 2003b. Color image processing using logarithmic operations. In: Proceedings of The IEEE International Symposium on Signals, Circuits and Systems, Iasi, Romania. pp. 133-136.

Pinoli, J.-C., 1992. Metrics, scalar product and correlation adapted to logarithmic images. Acta Stereol. 11, 157-168.

Pumo, B., Dhorne, T., 1998. Modèle logarithmique de régression optique. application à l'identification des fonctions de transmittance. Revue de statistique appliquée 46 (3), 65-75.

Schanda, J., 2007. Understanding the CIE System. Wiley-Interscience.

Zaharescu, E., 2003. Extending mathematical morphology for color images and for logarithmic representation of images. Ph.D. thesis, "Politehnica" University of Bucharest. 


\section{Main notations}

$\uparrow$ LIP addition

$\triangle$ LIP subtraction

$\triangle$ LIP multiplication

$F, G$ grey levels images

$D$ definition domain of images

$\alpha, \beta$ scalars elements

$i, j, k$ integer elements

$M$ maximum value of an image

$\mathcal{I}$ space of grey level images

$\mathcal{F}$ over-space of $\mathcal{I}$

$T_{F}$ transmittance of grey level image $F$

$C_{x, y}(F)$ physicians's contrast

$C_{R}, C_{G}, C_{B}$ observer correction constants $C_{x, y}^{\triangle}(F)$ LIP contrast

$d r(F)$ dynamic range of image $\mathrm{F}$
$\AA_{c}$ LIPC addition

$\triangle_{c}$ LIPC subtraction

$\otimes_{c}$ LIPC multiplication

$\mathbf{F}, \mathbf{G}, \mathbf{H}$ color images

$\mathrm{S}$ sensor sensitivity

$\alpha, \beta$ scalars elements

$i, j, k$ integer elements

$\mathbf{P}$ image perception by a sensor

$\mathbf{K}$ matrix such that $\mathbf{P}=\mathbf{K F}$

$\mathrm{X}, \mathrm{Y}$ normalisation matrices

$\mathbf{K}$ normalized matrix from $\mathbf{K}$

$\mathbf{P}$ image perception by a sensor

$\mathbf{U}$ matrix such that $\mathbf{P}=\mathbf{U T}$

Ú normalized matrix from $\mathbf{U}$

$\mathcal{I}^{3}$ space of color images

$\mathcal{F}^{3}$ over-space of $\mathcal{I}^{3}$

$\mathbf{T}_{\mathbf{F}}$ transmittance of color image $\mathbf{F}$ $C_{x, y}^{\triangle_{c}}(\mathbf{F})$ LIPC contrast

\section{Appendices}

\section{A. Image multiplication demonstration}

$$
\forall \mathbf{F} \in \mathcal{I}^{3}:
$$

$$
\begin{aligned}
& 2 \widehat{\otimes}_{C} \mathbf{F}=\mathbf{F} \wedge_{C} \mathbf{F}=\mathbf{K}^{-1} \mathbf{U}\left(\mathbf{U}^{-1} \mathbf{K} \mathbf{F}\right)^{2} \\
& 3 \otimes_{c} \mathbf{F}=\mathbf{F} \star_{c}\left(2 \otimes_{c} \mathbf{F}\right) \\
& \left.3 \otimes_{c} \mathbf{F}=\mathbf{K}^{-1} \mathbf{U}^{\prime} \mathbf{U}^{-1} \mathbf{K} \mathbf{F} * \mathbf{U}^{-1} \mathbf{K}^{\prime} \mathbf{K}^{-1} \mathbf{U}^{\prime}\left(\mathbf{U}^{-1} \mathbf{K} \mathbf{F}\right)^{2}\right] \\
& 3 \otimes_{c} \mathbf{F}=\mathbf{K}^{-1} \mathbf{U}^{\prime}\left(\mathbf{U}^{-1} \mathbf{K} \mathbf{F}\right)^{3}
\end{aligned}
$$

We suppose $\forall n \in \mathbb{N}^{+}$:

$$
n \otimes_{c} \mathbf{F}=\hat{\mathbf{K}}_{31}^{-1} \mathbf{U}^{\prime}\left(\mathbf{U}^{-1} \mathbf{K} \mathbf{F}\right)^{n}
$$


One can show that $(n+1) \otimes_{c} \mathbf{F}=\mathbf{K}^{-1} \mathbf{U}^{\prime}\left(\mathbf{K}^{-1} \mathbf{U} \mathbf{F}\right)^{n+1}$

$$
\begin{aligned}
& (n+1) \otimes_{c} \mathbf{F}=\mathbf{F} \star_{c}\left(n \otimes_{c} \mathbf{F}\right) \\
& (n+1) \otimes_{c} \mathbf{F}=\mathbf{K}^{-1} \mathbf{U}\left[\mathbf{U}^{-1} \mathbf{K} \mathbf{F} * \mathbf{U}^{-1} \mathbf{K}^{\prime}{ }^{-1} \mathbf{U}\left(\mathbf{U}^{-1} \mathbf{K} \mathbf{F}\right)^{n}\right] \\
& (n+1) \otimes_{c} \mathbf{F}=\mathbf{K}^{-1} \mathbf{U}\left(\mathbf{U}^{-1} \mathbf{K} \mathbf{F}\right)^{n+1}
\end{aligned}
$$

And finally $\forall n \in \mathbb{N}^{+}$:

$$
n \otimes_{c} \mathbf{F}=\hat{\mathbf{K}}^{-1} \mathbf{U}^{\prime}\left(\mathbf{U}^{-1} \mathbf{K} \mathbf{F}\right)^{n}
$$

The last expression is extended to each quotient of integer by proving $\forall \mathbf{F}, \mathbf{G} \in \mathcal{I}^{3}$ and $\forall p \in \mathbb{N}^{+}, \mathbf{F}=\frac{1}{p} \otimes_{c} \mathbf{G}$ verifies the expression $p \otimes_{c} \mathbf{F}=\mathbf{G}$. Indeed :

$$
\begin{aligned}
& p \otimes_{c} \mathbf{F}=\mathbf{G} \\
& \Longleftrightarrow \mathbf{K}^{-1} \mathbf{U}^{\prime}\left(\mathbf{U}^{-1} \mathbf{K} \mathbf{F}\right)^{p}=\mathbf{G} \\
& \Longleftrightarrow\left(\mathbf{U}^{-1} \mathbf{K} \mathbf{F}\right)^{p}=\mathbf{U}^{-1} \mathbf{K} \mathbf{G} \\
& \Longleftrightarrow \mathbf{U}^{-1} \mathbf{K} \mathbf{F}=\left(\mathbf{U}^{-1} \mathbf{K} \mathbf{G}\right)^{\frac{1}{p}} \\
& \Longleftrightarrow \mathbf{F}=\mathbf{K}^{-1} \mathbf{U}^{\prime}\left(\mathbf{U}^{-1} \mathbf{K} \mathbf{G}\right)^{\frac{1}{p}} \\
& \Longleftrightarrow \mathbf{F}=\frac{1}{p} \otimes_{c} \mathbf{G}
\end{aligned}
$$

Next, $\forall p \in \mathbb{N}, \forall q \in \mathbb{N}^{+}, \alpha \widehat{凶}_{c} \mathbf{F}$ is defined $\forall \alpha \in \mathbb{R}^{+}$as

$$
\begin{aligned}
\alpha \otimes_{c} \mathbf{F} & =\left(\lim _{n \rightarrow \infty} \frac{p_{n}}{q_{n}}\right) \otimes_{c} \mathbf{F} \\
\alpha \otimes_{c} \mathbf{F} & =\lim _{n \rightarrow \infty}\left(\frac{p_{n}}{q_{n}} \otimes_{c} \mathbf{F}\right) \\
\alpha \otimes_{c} \mathbf{F} & =\lim _{n \rightarrow \infty}\left[\dot{\mathbf{K}}^{-1} \mathbf{U}\left(\dot{\mathbf{U}}^{-1} \mathbf{K} \mathbf{F}\right)^{\frac{p_{n}}{q_{n}}}\right] \\
\alpha \otimes_{c} \mathbf{F} & =\mathbf{K}^{-1} \mathbf{U}\left(\dot{\mathbf{U}}^{-1} \mathbf{K} \mathbf{F}\right)^{\alpha} \text { with } \alpha=\lim _{n \rightarrow \infty} \frac{p_{n}}{q_{n}}
\end{aligned}
$$


B. Associativity of LIPC addition

$\forall \mathbf{F}, \mathbf{G}, \mathbf{H} \in \mathcal{I}^{3}$

$$
\begin{aligned}
& \mathbf{F} \AA_{c}\left(\mathbf{G} \AA_{c} \mathbf{H}\right)=\mathbf{K}^{-1} \mathbf{U}\left(\mathbf{U}^{-1} \mathbf{K} \mathbf{F} * \mathbf{U}^{-1} \mathbf{K} .\left(\mathbf{K}^{-1} \mathbf{U}\left(\mathbf{U}^{-1} \mathbf{K} \mathbf{G} * \mathbf{U}^{-1} \mathbf{K} . \mathbf{H}\right)\right)\right) \\
& \mathbf{F} \AA_{c}\left(\mathbf{G} \star_{c} \mathbf{H}\right)=\mathbf{K}^{-1} \mathbf{U}\left(\mathbf{U}^{-1} \mathbf{K} \mathbf{F} * \mathbf{U}^{-1} \mathbf{K} \mathbf{G} * \mathbf{U}^{-1} \hat{\mathbf{K}} . \mathbf{H}\right) \\
& \mathbf{F} \AA_{c}\left(\mathbf{G} \star_{c} \mathbf{H}\right)=\mathbf{K}^{-1} \mathbf{U}\left(\mathbf{U}^{-1} \mathbf{K}\left(\dot{\mathbf{K}}^{-1} \mathbf{U}^{\prime}\left(\mathbf{U}^{-1} \mathbf{K} \mathbf{F} * \mathbf{U}^{-1} \mathbf{K} \mathbf{G}\right)\right) * \mathbf{U}^{-1} \mathbf{K} . \mathbf{G}\right) \\
& \mathbf{F} \star_{c}\left(\mathbf{G} \star_{c} \mathbf{H}\right)=\left(\mathbf{F} \star_{c} \mathbf{G}\right) \star_{c} \mathbf{H}
\end{aligned}
$$

C. Distributivity of scalar addition with respect to LIPC multiplication

$$
\begin{aligned}
& \forall \alpha, \beta \in \mathbb{R}^{+}, \forall \mathbf{F} \in \mathcal{I}^{3} \\
& (\alpha+\beta) \otimes_{c} \mathbf{F}=\dot{\mathbf{K}}^{-1} \mathbf{U}\left(\mathbf{U}^{-1} \mathbf{K} \mathbf{F}\right)^{\alpha+\beta} \\
& \left(\alpha \otimes_{c} \mathbf{F}\right) \AA_{c}\left(\beta \otimes_{c} \mathbf{F}\right)=\mathbf{K}^{-1} \mathbf{U}^{\prime}\left(\mathbf{U}^{-1} \mathbf{K}\left[\hat{\mathbf{K}}^{-1} \mathbf{U}\left(\mathbf{U}^{-1} \mathbf{K} \mathbf{F}\right)^{\alpha}\right] * \mathbf{U}^{-1} \mathbf{K}^{\prime}\left[\dot{\mathbf{K}}^{-1} \mathbf{U}^{\prime}\left(\mathbf{U}^{-1} \mathbf{K} \mathbf{F}\right)^{\beta}\right]\right) \\
& \left(\alpha \otimes_{c} \mathbf{F}\right) \triangle_{c}\left(\beta \otimes_{c} \mathbf{F}\right)=\mathbf{K}^{-1} \mathbf{U}^{\prime}\left(\mathbf{U}^{-1} \mathbf{K} \mathbf{F}\right)^{\alpha+\beta} \\
& \left(\alpha \otimes_{c} \mathbf{F}\right) \AA_{c}\left(\beta \otimes_{c} \mathbf{F}\right)=(\alpha+\beta) \otimes_{c} \mathbf{F}
\end{aligned}
$$


D. Distributivity of LIPC scalar multiplication with respect to LIPC addition

$$
\begin{aligned}
& \forall \alpha \in \mathbb{R}^{+}, \forall \mathbf{F}, \mathbf{G} \in \mathcal{I}^{3} \\
& \alpha \widehat{\triangle}_{c}\left(\mathbf{F} \AA_{c} \mathbf{G}\right)= \\
& =\mathbf{K}^{-1} \mathbf{U}\left[\mathbf{U}^{-1} \mathbf{K}\left(\mathbf{K}^{-1} \mathbf{U}\left(\mathbf{U}^{-1} \mathbf{K} \mathbf{F} * \mathbf{U}^{-1} \mathbf{K} \mathbf{G}\right)\right)\right]^{\alpha} \\
& =\dot{\mathbf{K}}^{-1} \mathbf{U}\left(\mathbf{U}^{-1} \mathbf{K} \mathbf{F} * \mathbf{U}^{-1} \mathbf{K} \mathbf{G}\right)^{\alpha} \\
& =\mathbf{K}^{-1} \mathbf{U}\left[\left(\mathbf{U}^{-1} \mathbf{K} \mathbf{F}\right)^{\alpha} *\left(\mathbf{U}^{-1} \mathbf{K} \mathbf{G}\right)^{\alpha}\right] \\
& =\mathbf{K}^{-1} \mathbf{U}\left[\mathbf{U}^{-1} \mathbf{K}^{\prime}{ }^{-1} \mathbf{U}\left(\mathbf{U}^{-1} \mathbf{K} \mathbf{F}\right)^{\alpha} * \mathbf{U}^{-1} \mathbf{K}^{\prime}{ }^{-1} \mathbf{U}^{\prime}\left(\mathbf{U}^{-1} \mathbf{K} \mathbf{G}\right)^{\alpha}\right] \\
& =\left(\alpha \otimes_{c} \mathbf{F}\right) \star_{c}\left(\alpha \otimes_{c} \mathbf{G}\right)
\end{aligned}
$$

E. Associativity of LIPC multiplication

$$
\begin{aligned}
& \forall \alpha, \beta \in \mathbb{R}^{+}, \forall \mathbf{F}, \mathbf{G} \in \mathcal{I}^{3}: \\
& (\alpha \times \beta) \otimes_{c} \mathbf{F}=\dot{\mathbf{K}}^{-1} \mathbf{U}\left(\mathbf{U}^{-1} \mathbf{K} \mathbf{F}\right)^{j k} \\
& \begin{aligned}
\alpha \otimes_{c}\left(\beta \otimes_{c} \mathbf{F}\right) & =\dot{\mathbf{K}}^{-1} \mathbf{U}^{\prime}\left[\mathbf{U}^{-1} \mathbf{K}^{-1} \mathbf{U}^{-1}\left(\mathbf{U}^{-1} \mathbf{K} \mathbf{F}\right)^{\beta}\right]^{\alpha} \\
& =\dot{\mathbf{K}}^{-1} \mathbf{U}^{\prime}\left(\mathbf{U}^{-1} \mathbf{K} \mathbf{F}\right)^{j k} \\
& =(\alpha \times \beta) \otimes_{c} \mathbf{F}
\end{aligned}
\end{aligned}
$$




\section{F. Ten degrees RGB Color Matching Function of Stiles and Burch (1959)}

From http://cvrl.ucl.ac.uk/

\begin{tabular}{|c|c|c|c|c|c|c|c|}
\hline Wavelength(nm) & $\mathrm{r}$ & $\mathrm{g}$ & $\mathrm{b}$ & Wavelength$(\mathrm{nm})$ & $\mathrm{r}$ & $\mathrm{g}$ & $\mathrm{b}$ \\
\hline 390 & 0,00 & 0,00 & 0,01 & 480 & $-0,38$ & 0,34 & 0,35 \\
\hline 395 & 0,00 & 0,00 & 0,02 & 485 & $-0,41$ & 0,41 & 0,26 \\
\hline 400 & 0,01 & 0,00 & 0,04 & 490 & $-0,43$ & 0,47 & 0,18 \\
\hline 405 & 0,02 & $-0,01$ & 0,09 & 495 & $-0,45$ & 0,55 & 0,13 \\
\hline 410 & 0,04 & $-0,01$ & 0,18 & 500 & $-0,44$ & 0,63 & 0,09 \\
\hline 415 & 0,05 & $-0,02$ & 0,31 & 505 & $-0,41$ & 0,71 & 0,06 \\
\hline 420 & 0,07 & $-0,03$ & 0,47 & 510 & $-0,37$ & 0,79 & 0,04 \\
\hline 425 & 0,08 & $-0,03$ & 0,62 & 515 & $-0,28$ & 0,87 & 0,02 \\
\hline 430 & 0,07 & $-0,03$ & 0,76 & 520 & $-0,19$ & 0,95 & 0,01 \\
\hline 435 & 0,06 & $-0,03$ & 0,88 & 525 & $-0,04$ & 0,99 & 0,00 \\
\hline 440 & 0,03 & $-0,02$ & 0,98 & 530 & 0,13 & 1,02 & 0,00 \\
\hline 445 & 0,00 & 0,00 & 1,00 & 535 & 0,31 & 1,04 & $-0,01$ \\
\hline 450 & $-0,05$ & 0,03 & 1,00 & 540 & 0,54 & 1,05 & $-0,01$ \\
\hline 455 & $-0,10$ & 0,06 & 0,91 & 545 & 0,77 & 1,04 & $-0,01$ \\
\hline 460 & $-0,16$ & 0,11 & 0,83 & 550 & 1,01 & 1,00 & $-0,01$ \\
\hline 465 & $-0,22$ & 0,16 & 0,74 & 555 & 1,27 & 0,97 & $-0,01$ \\
\hline 470 & $-0,28$ & 0,22 & 0,61 & 560 & 1,56 & 0,92 & $-0,01$ \\
\hline 475 & $-0,33$ & 0,28 & 0,47 & 565 & 1,85 & 0,86 & $-0,01$ \\
\hline
\end{tabular}

Table 3: Ten degrees RGB Color Matching Function of Stiles and Burch(1959) (from $390 \mathrm{~nm}$ to $475 \mathrm{~nm})$ 


\begin{tabular}{|c|c|c|c|c|c|c|c|}
\hline Wavelength(nm) & $\mathrm{r}$ & $\mathrm{g}$ & $\mathrm{b}$ & Wavelength(nm) & $\mathrm{r}$ & $\mathrm{g}$ & $\mathrm{b}$ \\
\hline 570 & 2,15 & 0,78 & $-0,01$ & 675 & 0,17 & 0,00 & 0,00 \\
\hline 575 & 2,43 & 0,70 & $-0,01$ & 680 & 0,12 & 0,00 & 0,00 \\
\hline 580 & 2,66 & 0,60 & $-0,01$ & 685 & 0,09 & 0,00 & 0,00 \\
\hline 585 & 2,92 & 0,51 & $-0,01$ & 690 & 0,06 & 0,00 & 0,00 \\
\hline 590 & 3,08 & 0,42 & 0,00 & 695 & 0,04 & 0,00 & 0,00 \\
\hline 595 & 3,16 & 0,34 & 0,00 & 700 & 0,03 & 0,00 & 0,00 \\
\hline 600 & 3,17 & 0,26 & 0,00 & 705 & 0,02 & 0,00 & 0,00 \\
\hline 605 & 3,10 & 0,19 & 0,00 & 710 & 0,01 & 0,00 & 0,00 \\
\hline 610 & 2,95 & 0,14 & 0,00 & 715 & 0,01 & 0,00 & 0,00 \\
\hline 615 & 2,72 & 0,09 & 0,00 & 720 & 0,01 & 0,00 & 0,00 \\
\hline 620 & 2,45 & 0,06 & 0,00 & 725 & 0,00 & 0,00 & 0,00 \\
\hline 625 & 2,17 & 0,04 & 0,00 & 730 & 0,00 & 0,00 & 0,00 \\
\hline 630 & 1,84 & 0,02 & 0,00 & 735 & 0,00 & 0,00 & 0,00 \\
\hline 635 & 1,52 & 0,01 & 0,00 & 740 & 0,00 & 0,00 & 0,00 \\
\hline 640 & 1,24 & 0,00 & 0,00 & 745 & 0,00 & 0,00 & 0,00 \\
\hline 645 & 1,01 & 0,00 & 0,00 & 750 & 0,00 & 0,00 & 0,00 \\
\hline 650 & 0,78 & 0,00 & 0,00 & 755 & 0,00 & 0,00 & 0,00 \\
\hline 655 & 0,59 & 0,00 & 0,00 & 760 & 0,00 & 0,00 & 0,00 \\
\hline 660 & 0,44 & 0,00 & 0,00 & 765 & 0,00 & 0,00 & 0,00 \\
\hline 665 & 0,33 & 0,00 & 0,00 & 770 & 0,00 & 0,00 & 0,00 \\
\hline 670 & 0,24 & 0,00 & 0,00 & 775 & 0,00 & 0,00 & 0,00 \\
\hline & & & & 780 & 0,00 & 0,00 & 0,00 \\
\hline
\end{tabular}

Table 4: Ten degrees RGB Color Matching Function of Stiles and Burch(1959) (from $570 \mathrm{~nm}$ to $780 \mathrm{~nm})$ 


\section{G. D65 power spectral distribution}

From http://cvrl.ucl.ac.uk/

\begin{tabular}{|c|c|c|c|}
\hline wavelength $(\mathrm{nm})$ & spectral distribution & wavelength $(\mathrm{nm})$ & spectral distribution \\
\hline 390 & 54,65 & 485 & 112,37 \\
\hline 395 & 68,70 & 490 & 108,81 \\
\hline 400 & 82,75 & 495 & 109,08 \\
\hline 405 & 87,12 & 500 & 109,35 \\
\hline 410 & 91,49 & 505 & 108,58 \\
\hline 415 & 92,46 & 510 & 107,80 \\
\hline 420 & 93,43 & 515 & 106,30 \\
\hline 425 & 90,06 & 520 & 104,79 \\
\hline 430 & 86,68 & 525 & 106,24 \\
\hline 435 & 95,77 & 530 & 107,69 \\
\hline 440 & 104,86 & 535 & 106,05 \\
\hline 445 & 110,94 & 540 & 104,41 \\
\hline 450 & 117,01 & 545 & 104,22 \\
\hline 455 & 117,41 & 550 & 104,05 \\
\hline 460 & 117,81 & 555 & 102,02 \\
\hline 465 & 116,34 & 560 & 100,00 \\
\hline 470 & 114,86 & 565 & 98,17 \\
\hline 475 & 115,39 & 570 & 96,33 \\
\hline 480 & 115,92 & 575 & 96,06 \\
\hline & & 580 & 95,79 \\
\hline
\end{tabular}

Table 5: CIE Standard Illuminant D65 relative spectralpower distribution (from 390nm to $580 \mathrm{~nm}$ ) 


\begin{tabular}{|c|c|c|c|}
\hline wavelength $(\mathrm{nm})$ & spectral distribution & wavelength $(\mathrm{nm})$ & spectral distribution \\
\hline 585 & 92,24 & 685 & 74,00 \\
\hline 590 & 88,69 & 690 & 69,72 \\
\hline 595 & 89,35 & 695 & 70,67 \\
\hline 600 & 90,01 & 700 & 71,61 \\
\hline 605 & 89,80 & 705 & 72,98 \\
\hline 610 & 89,60 & 710 & 74,35 \\
\hline 615 & 88,65 & 715 & 67,98 \\
\hline 620 & 87,70 & 720 & 61,60 \\
\hline 625 & 85,49 & 725 & 65,74 \\
\hline 630 & 83,29 & 730 & 69,89 \\
\hline 635 & 83,49 & 735 & 72,49 \\
\hline 640 & 83,70 & 740 & 75,09 \\
\hline 645 & 81,86 & 745 & 69,34 \\
\hline 650 & 80,03 & 750 & 63,59 \\
\hline 655 & 80,12 & 755 & 55,01 \\
\hline 660 & 80,21 & 760 & 46,42 \\
\hline 665 & 81,25 & 765 & 56,61 \\
\hline 670 & 82,28 & 770 & 66,81 \\
\hline 675 & 80,28 & 775 & 65,09 \\
\hline 680 & 78,28 & 780 & 63,38 \\
\hline
\end{tabular}

Table 6: CIE Standard Illuminant D65 relative spectralpower distribution (from 585nm to $780 \mathrm{~nm})$ 


\section{H. Figures}

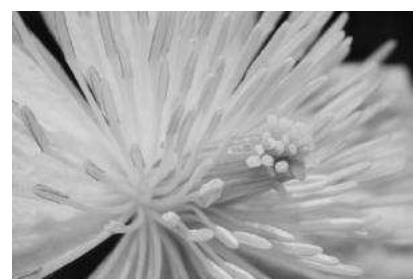

(a)

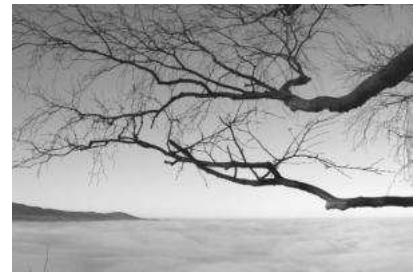

(b)

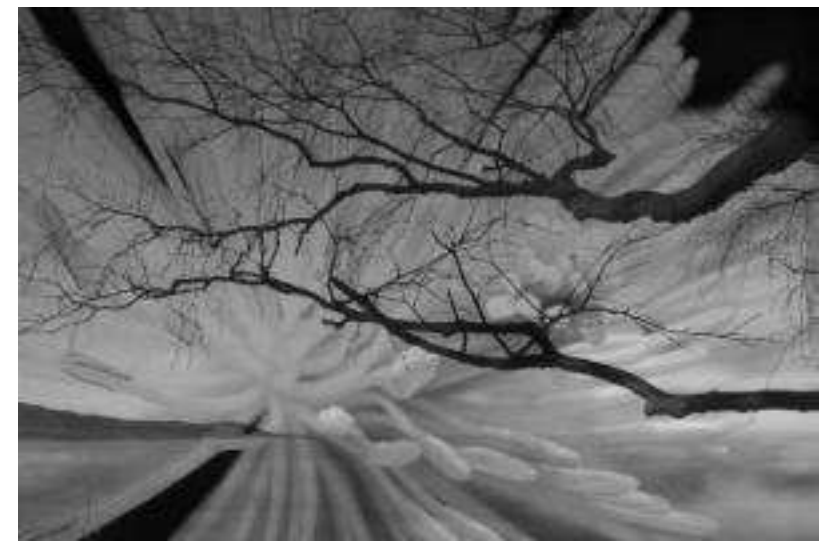

(c)

Figure 1: (a) and (b) initial grey level images, (c) LIP addition of (a) by (b)

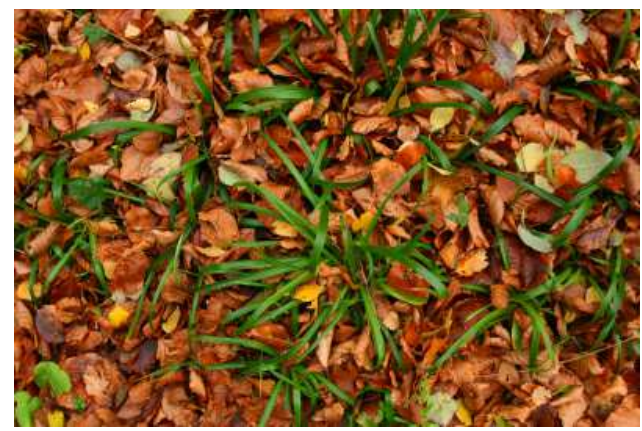

(a)

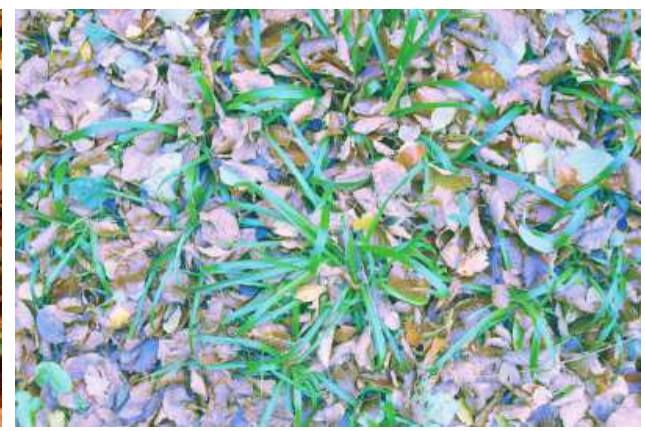

(b)

Figure 2: (a) initial image, (b) false color generation by grey level LIP multiplication on each channel, in order to maintain each mean channel value to 125 


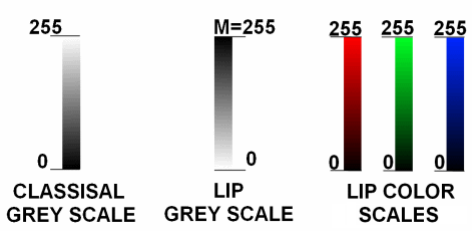

Figure 3: grey scales differences between LIP and classical

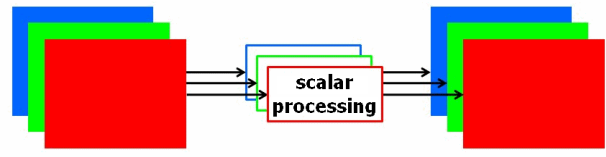

Figure 4: Scalar processing of an image

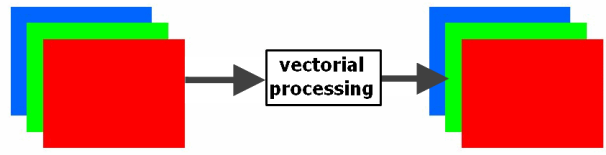

Figure 5: Vectorial processing of an image

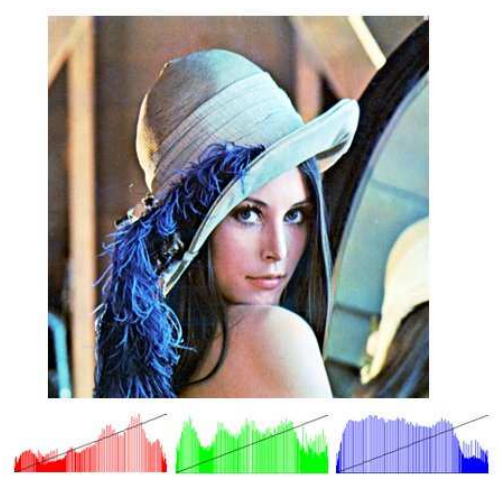

(a)

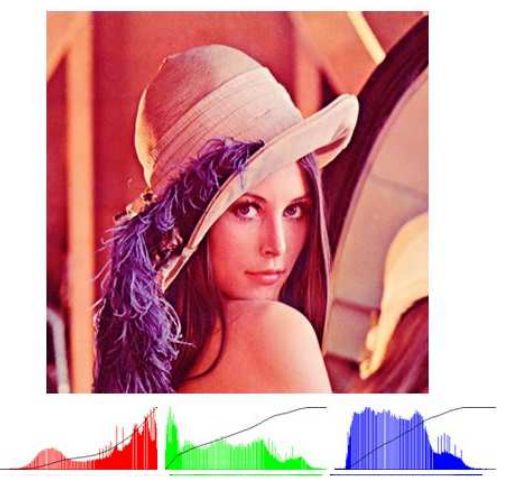

(b)

Figure 6: (a) scalar processing with histogram equalization on each channel, (b) vectorial treatment with histogram equalization on the entire data at the same time 


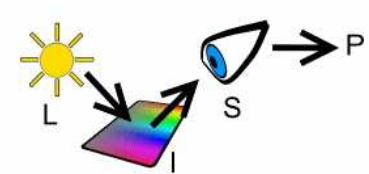

(a)

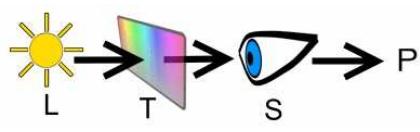

(b)

Figure 7: (a) perception of an image by the eye : image $\mathbf{I}$ lighted by $\mathbf{L}$ and viewed by $\mathbf{S}$, (b) sensor perception depending on the light $\mathbf{L}$, the transmittance $\mathbf{T}$ and the sensor $\mathbf{S}$

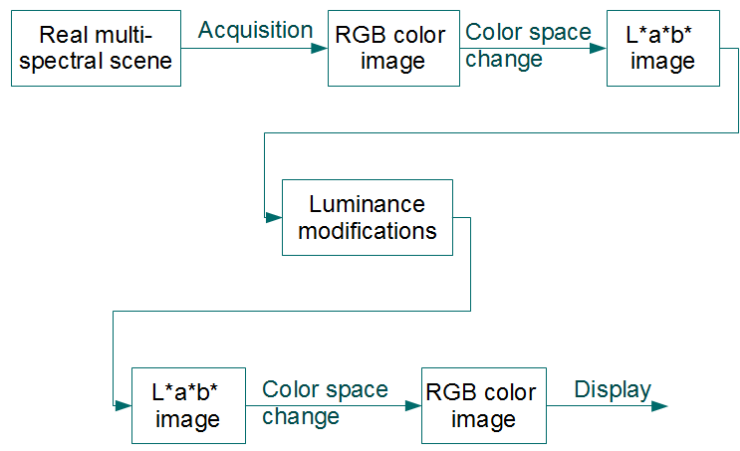

Figure 8: Common color algorithm

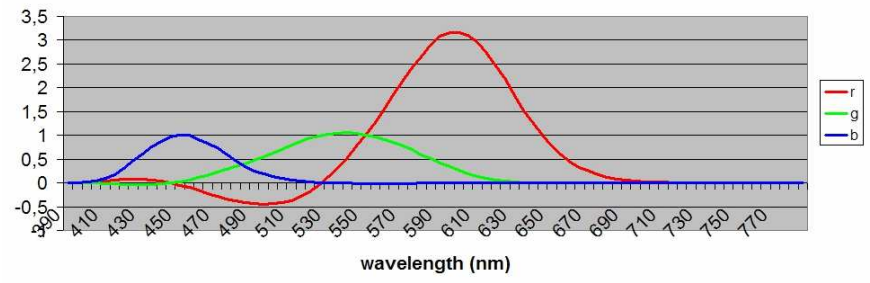

Figure 9: R, G and B color matching functions

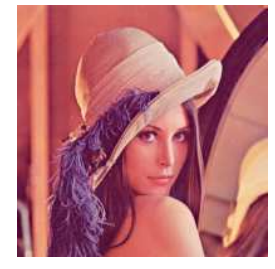

(a)

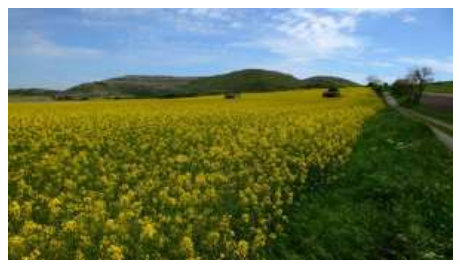

(b)

Figure 10: (a) image Lena (512x512 pixels), (b) : image P110 (1920x1080 pixels) 


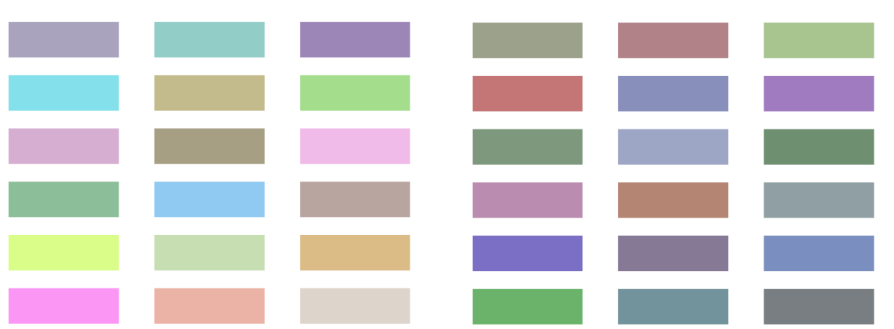

(a)
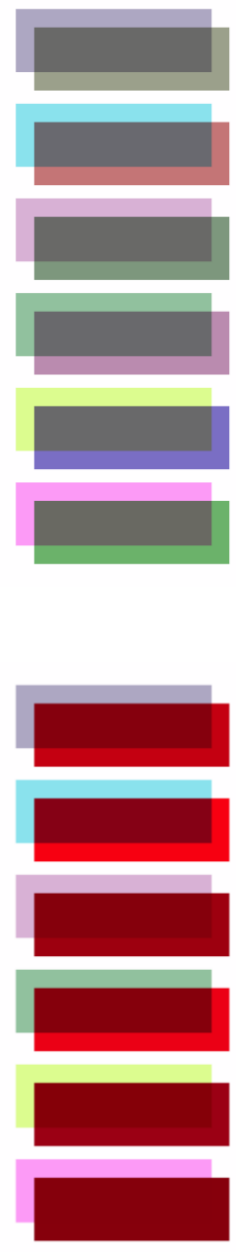

(b)
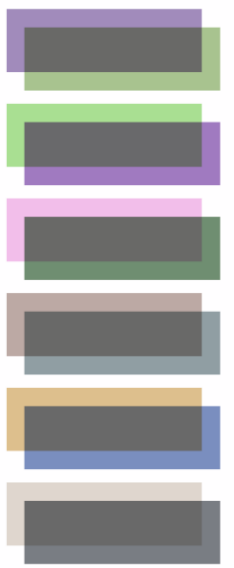

(c)
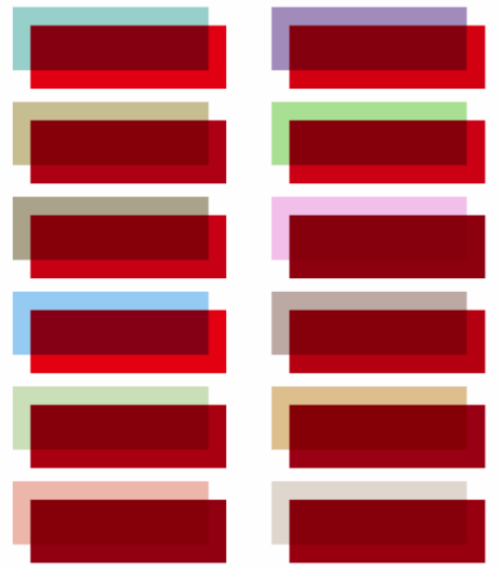

(d)

Figure 11: (a) original set of colors, (b) complementary set of colors to obtain a grey color, (c) Result of the LIPC addition of (a) and (b), (d) result of the LIPC addition when using a red complementary 


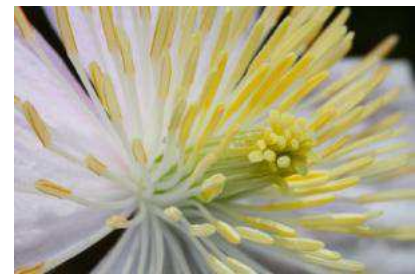

(a)

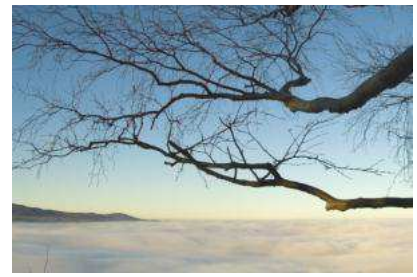

(b)

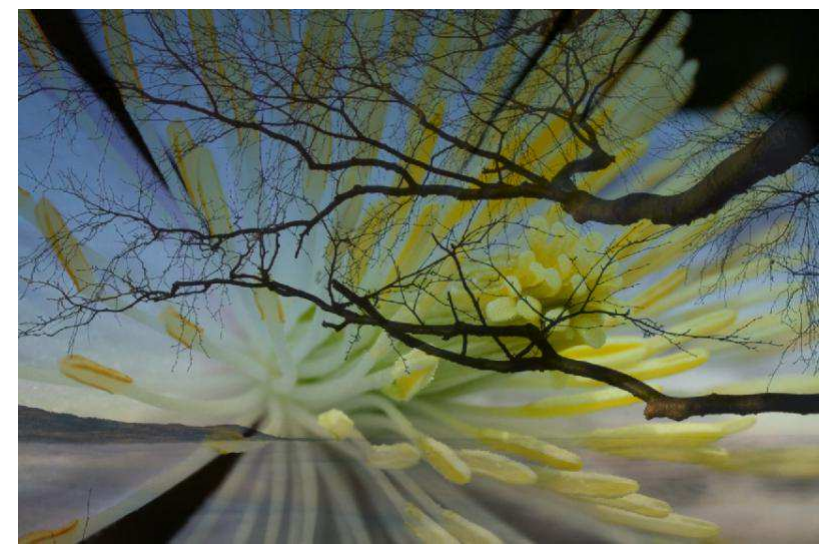

(c)

Figure 12: (a) and (b) initial images, (c) LIPC addition of (a) and (b)

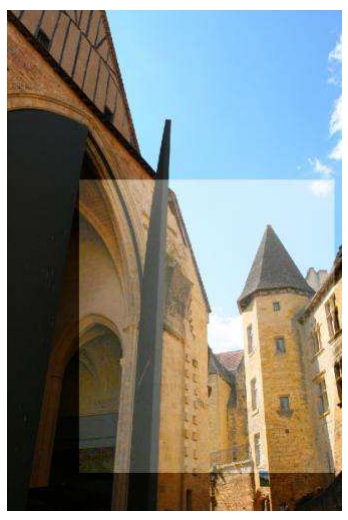

(a)

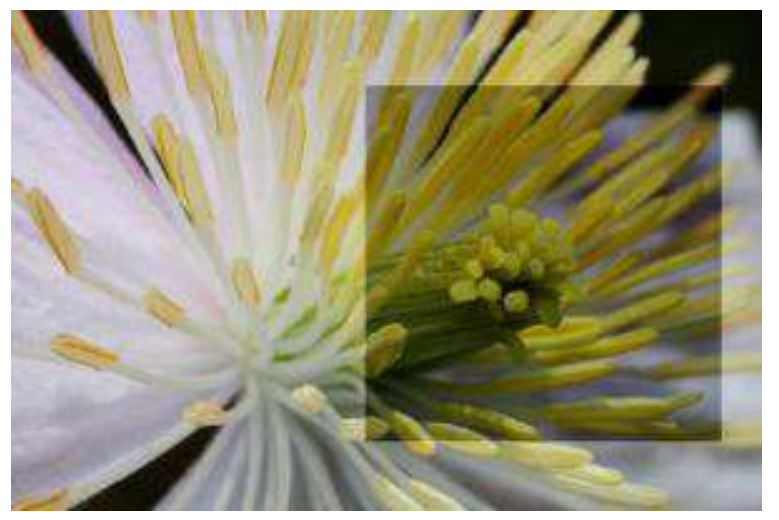

(b)

Figure 13: initial image with result of multiplication in superposition, (a) multiplication by 0.4 , (b) multiplication by 2.6 


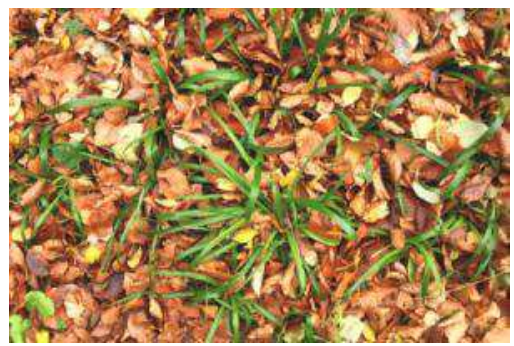

(a)

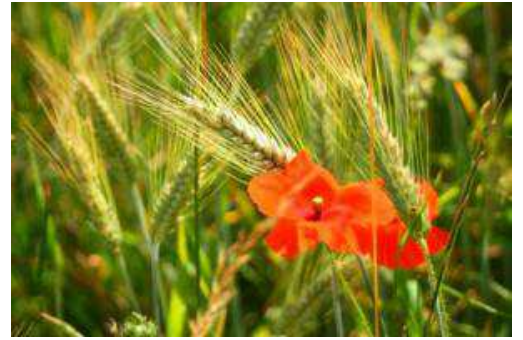

(b)

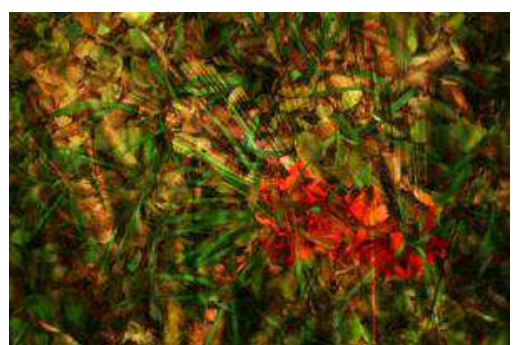

(c)

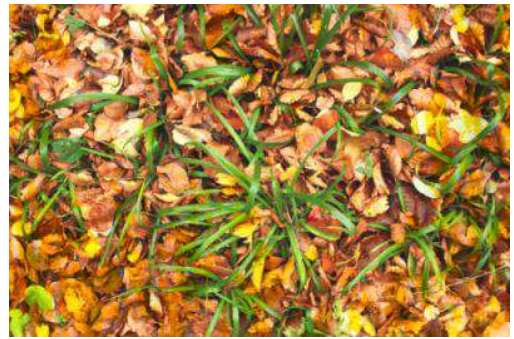

(d)

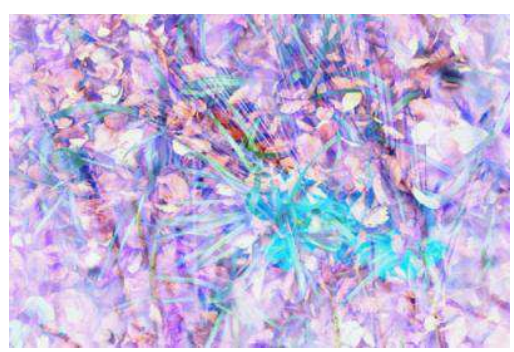

(e)

Figure 14: (a) and (b) initial images, (c) LIPC addition of (a) by (b), (d) LIPC subtraction of (c) by (b), (e) normalized result of classical subtraction of (c) by (b) 

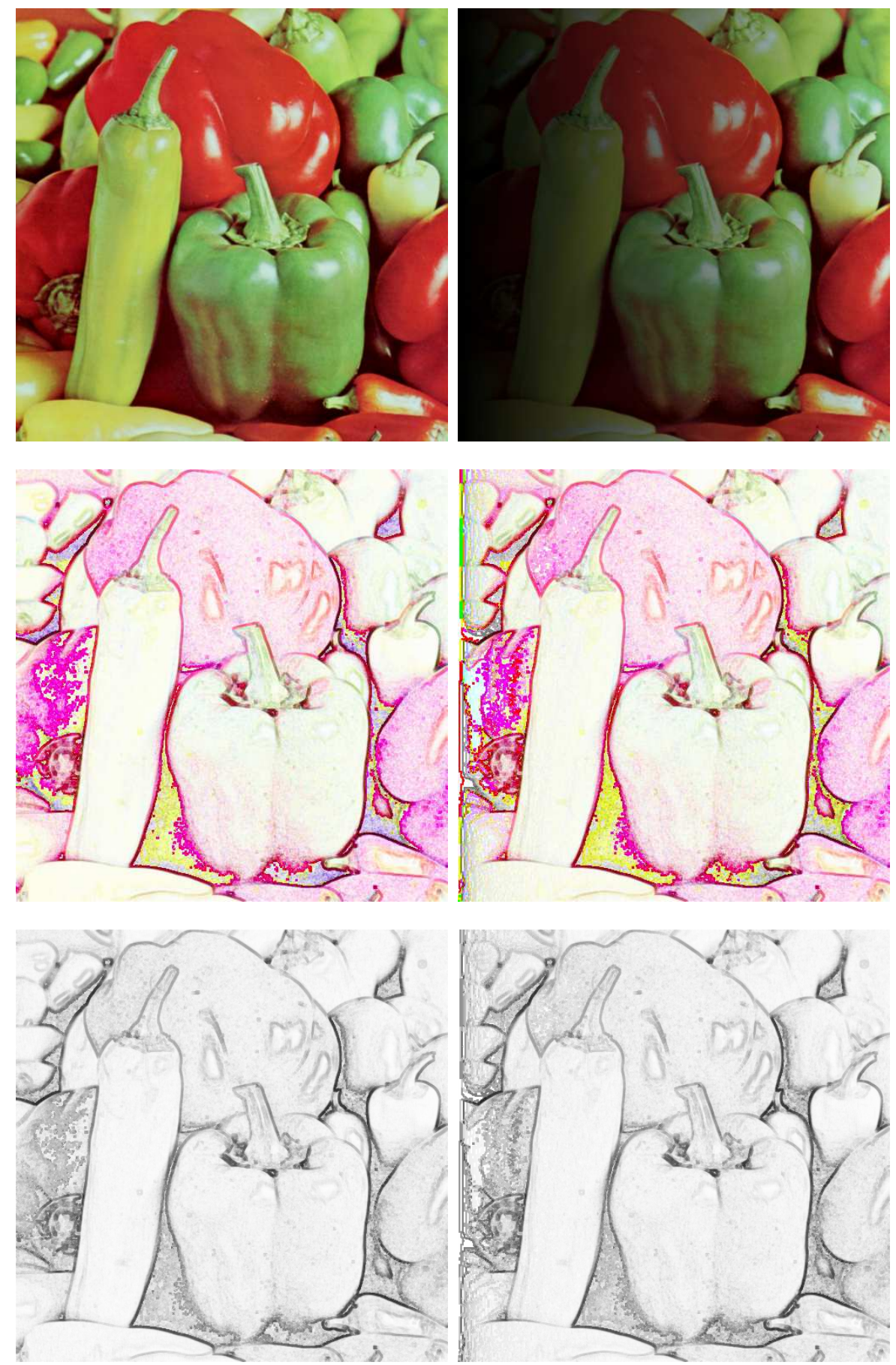

Figure 15: First row : initial peppers image (left) and darken peppers image (right), second row : corresponding contrast, third row : corresponding norm 


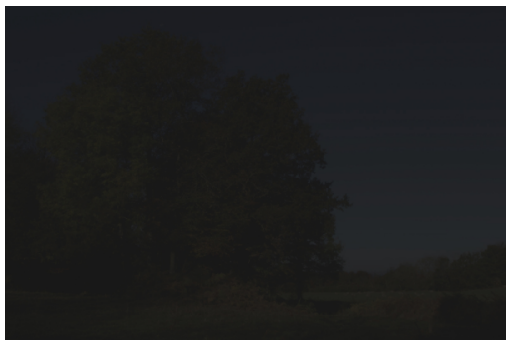

(a)

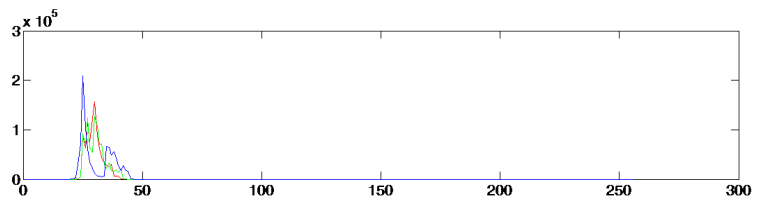

(c)

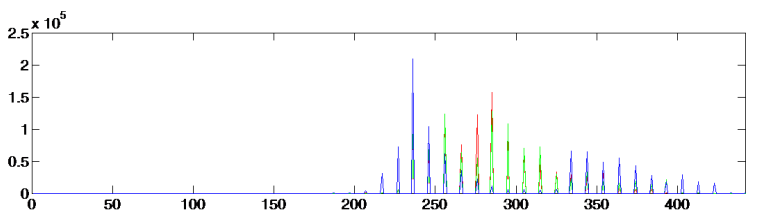

(d)

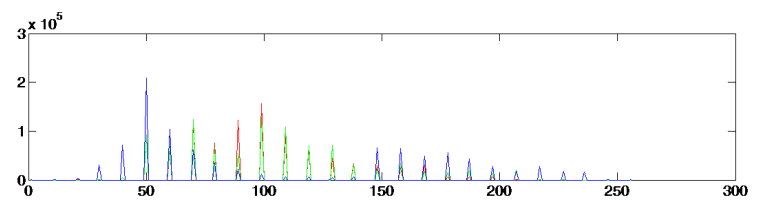

(e)

d)

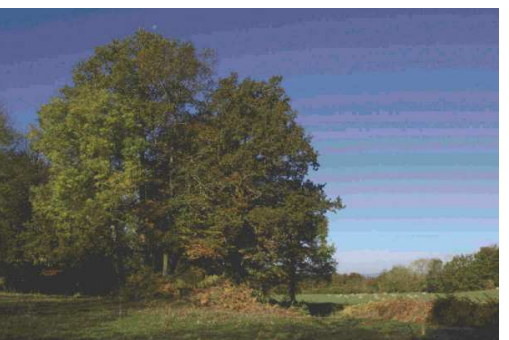

(b)

Figure 16: (a) : image taken with low lightening conditions, (b) : corrected LIPC subtraction of (a) by $\mathbf{C}_{O}$, (c) : histogram R, G and B of (a), (d) : histogram R, G and $\mathrm{B}$ of LIPC subtraction of (a) by $\mathbf{C}_{0},(\mathrm{e})$ histogram $\mathrm{R}, \mathrm{G}$ and $\mathrm{B}$ of $(c)-\operatorname{Inf}((c))$

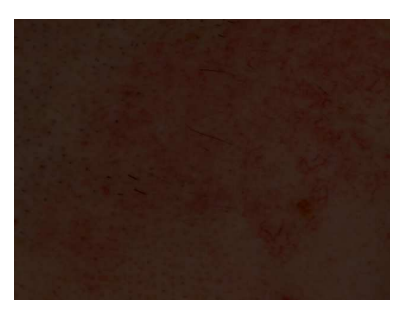

(c)

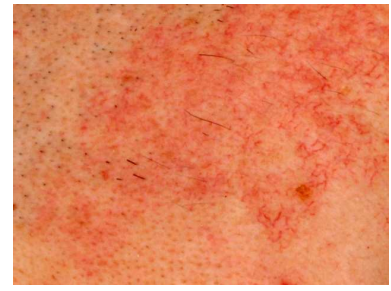

(d)

Figure 17: (a) initial image with $\operatorname{Inf}=0$, (b) LIP subtraction of (a) by $\operatorname{Sup}((\mathrm{a}))$ 


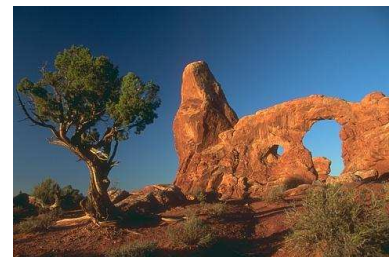

(a)

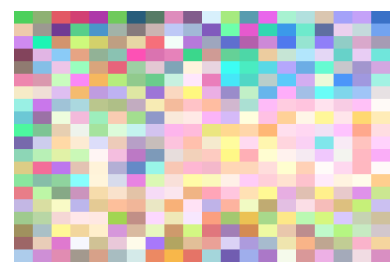

(b)

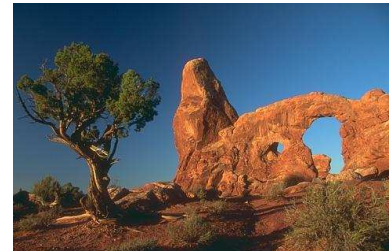

(d)

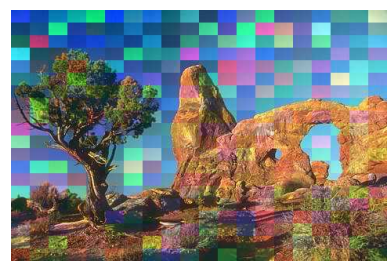

(c)

Figure 18: (a) initial image from Berkeley Segmentation Dataset and Benchmark (see Martin et al. (2001)), (b) and (c) complementary images of (a), LIPC addition of (b) and (c)

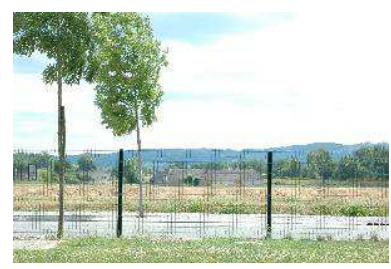

(a)

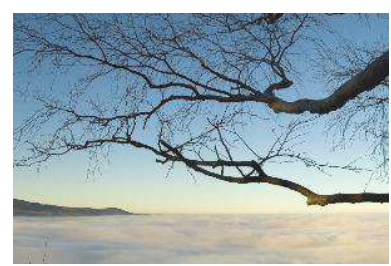

(b)
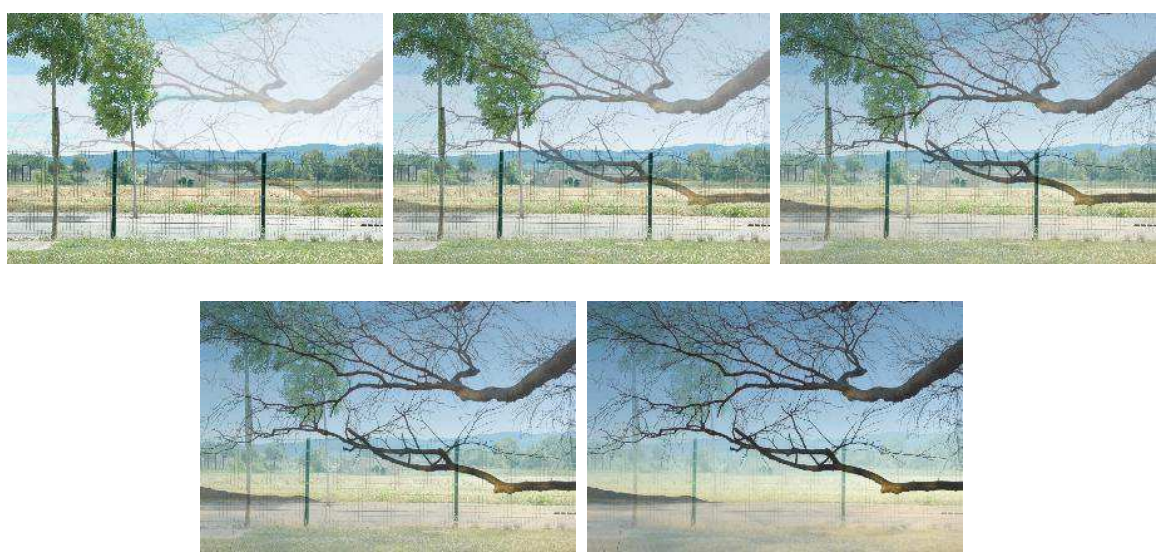

(c)

Figure 19: (a) and (b) initial images $(\mathrm{i}=6$ and $\mathrm{i}=0),(\mathrm{c})$ transition steps $(\mathrm{i}=5$ to to $\mathrm{i}=1)$ for $\mathrm{n}=6$ 


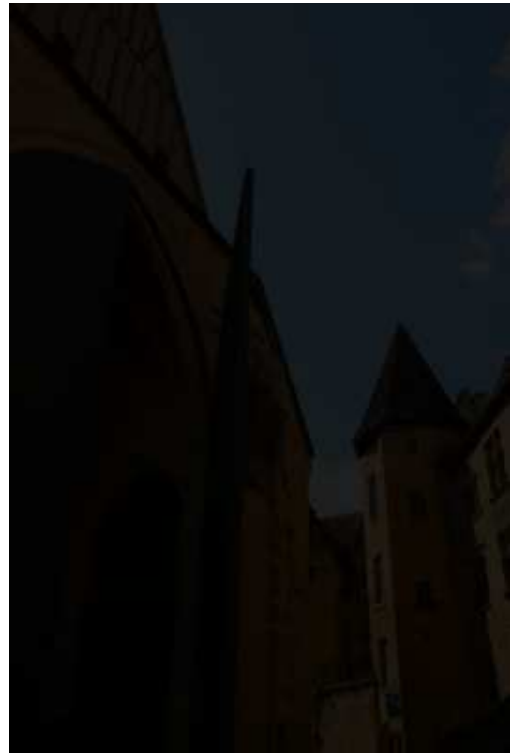

(a)

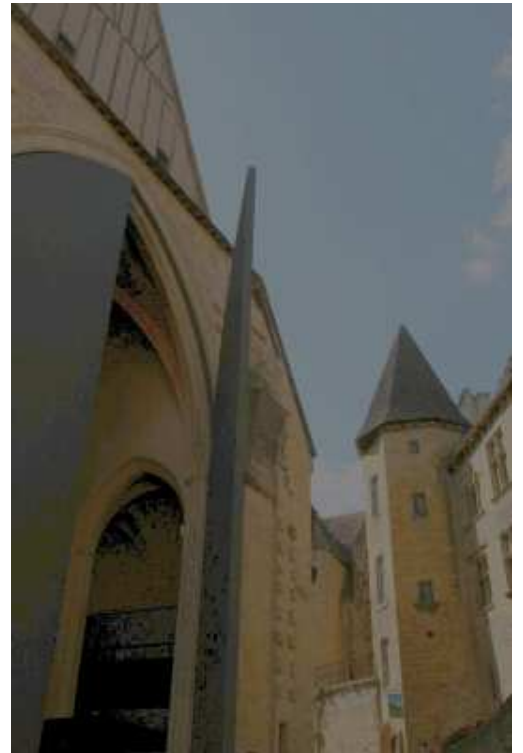

(b)

Figure 20: (a) initial image with low lighting conditions, (b) corrected image with dynamic centring by optimal $\lambda_{0}$

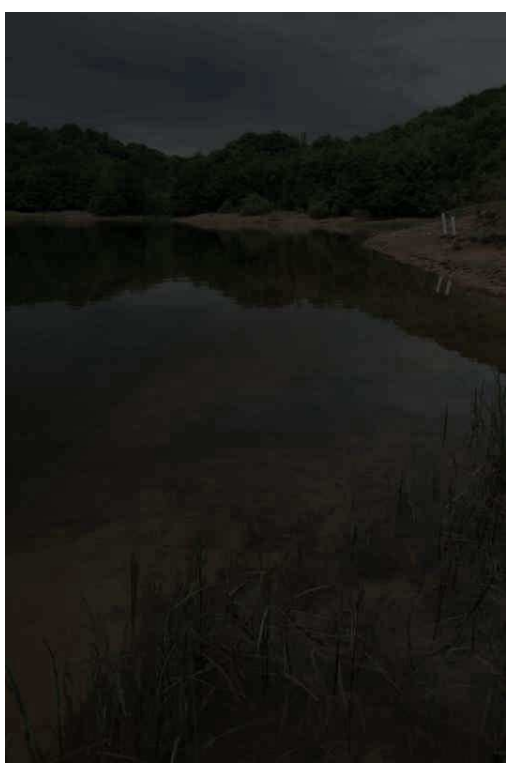

(a)

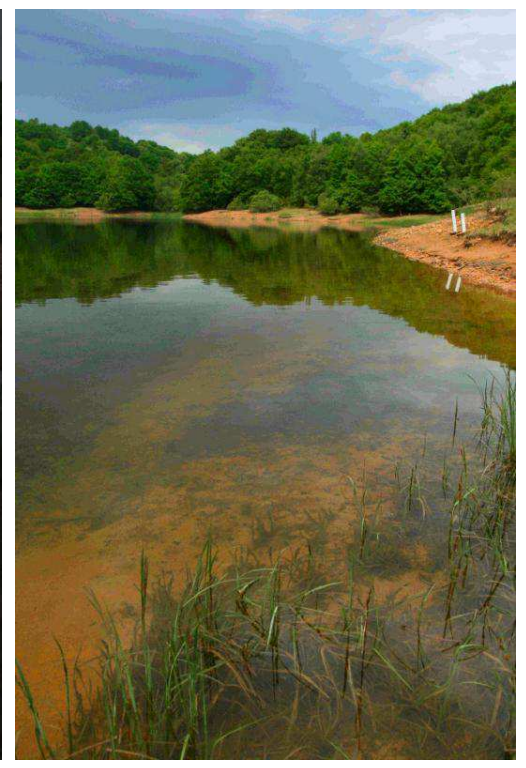

(b)

Figure 21: (a) initial image, (b) mean centring of initial image near to 90 

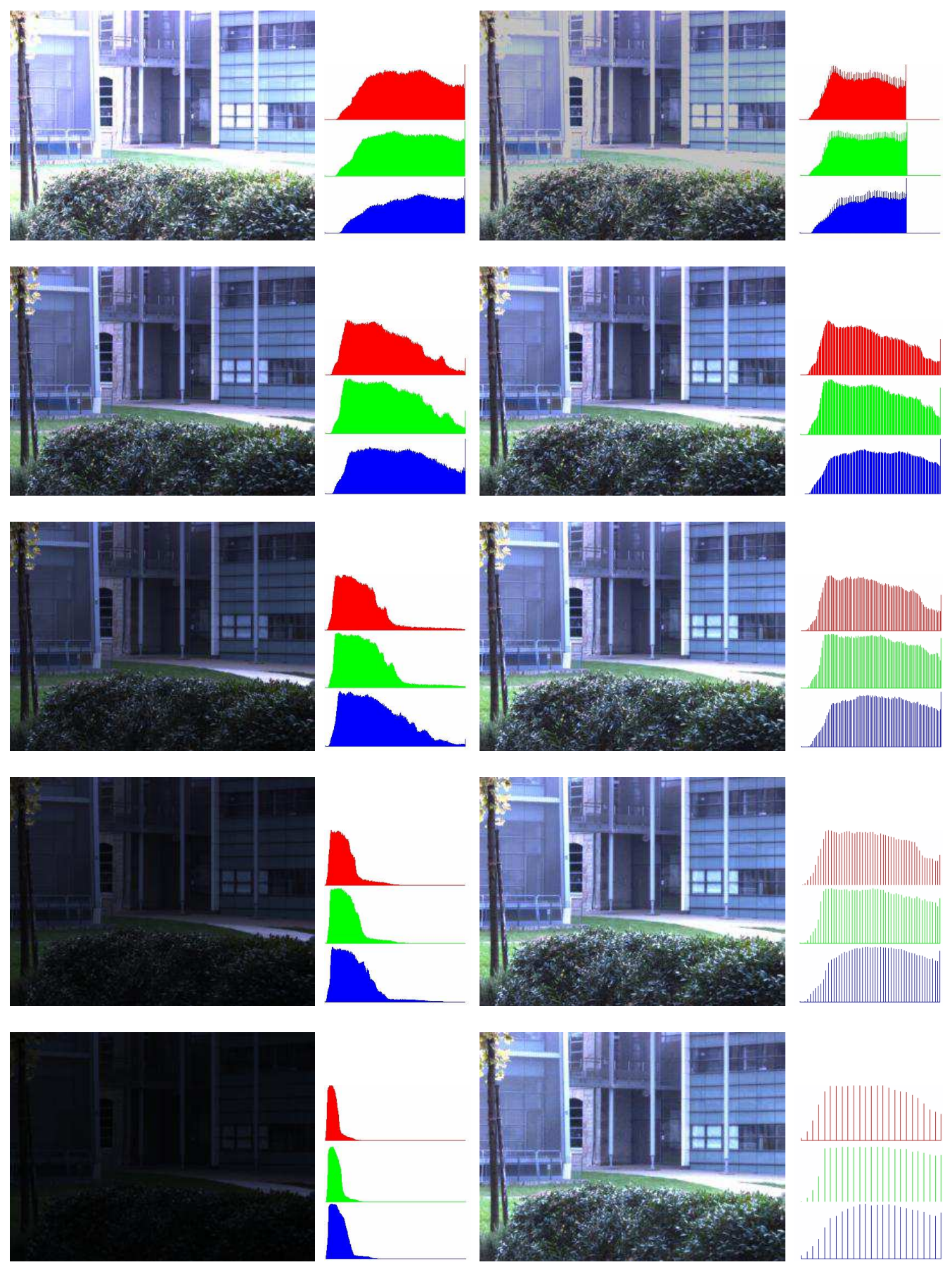

Figure 22: Left column : initial images and associated (R, G, B) histograms aperture values $(4,6,8,12,16)$, right column : corrected images to a constant mean value (125) and corresponding histograms 

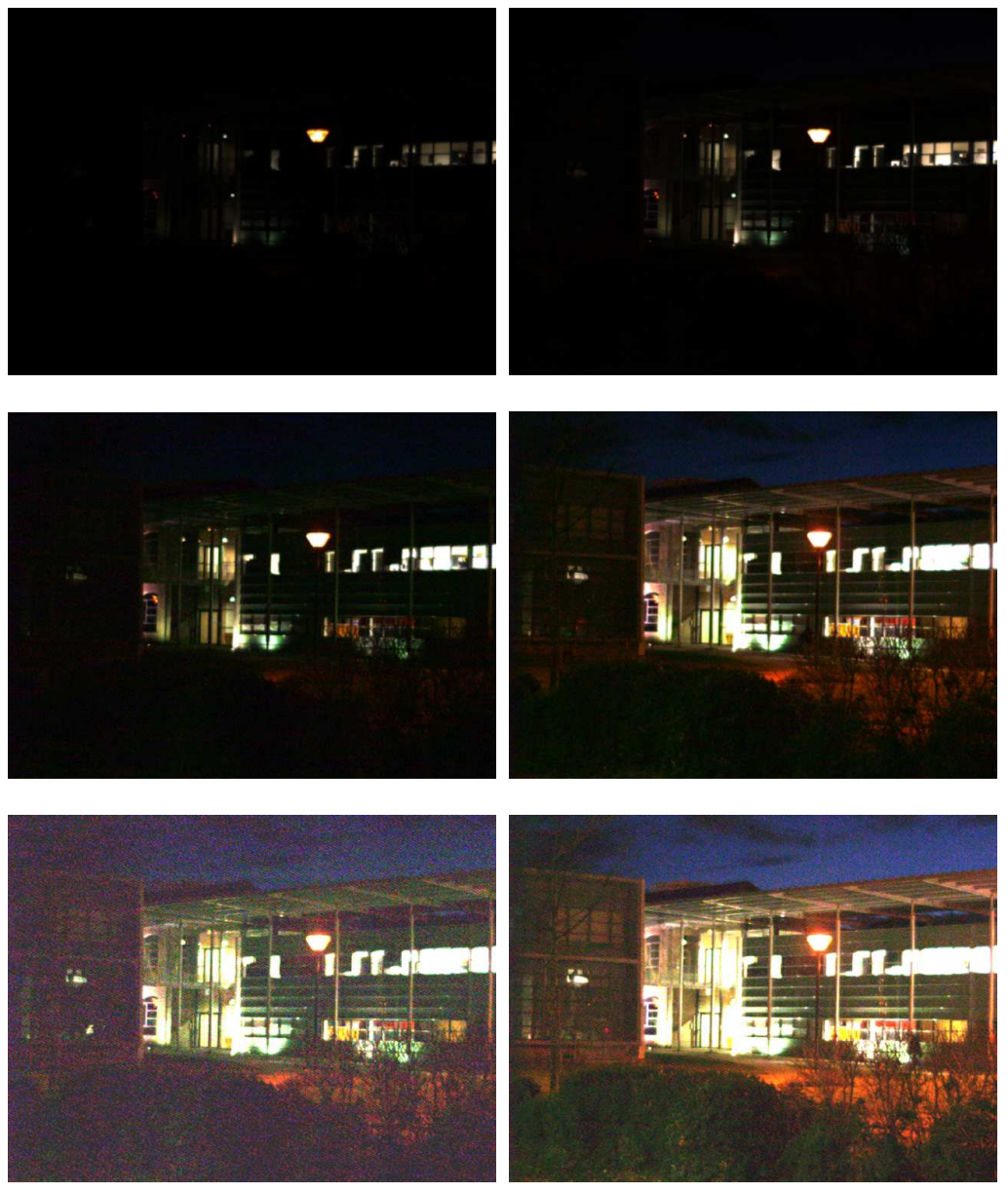

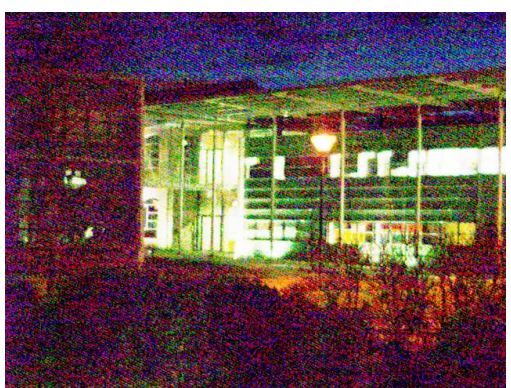

(a)

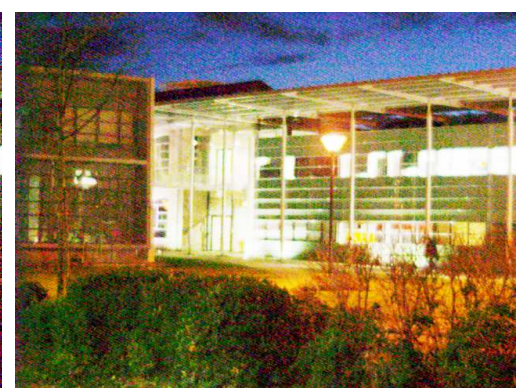

(b)

Figure 23: column (a) : image taken at $30 \mathrm{~ms}$ exposure time, column (b) : image taken at $100 \mathrm{~ms}$ exposure time, first row : intial images, second row : maximum gain augmentation, third row : LIPC subtraction by 250 , fourth row : histogram equalization 